\title{
Exploring sizable triple Higgs couplings in the 2HDM
}

\author{
F. Arco ${ }^{1,2, a}$, S. Heinemeyer ${ }^{2,3,4, b}$, M. J. Herrero ${ }^{1,2, c}$ \\ ${ }^{1}$ Departamento de Física Teórica, Universidad Autónoma de Madrid, Cantoblanco, 28049 Madrid, Spain \\ ${ }^{2}$ Instituto de Física Teórica (UAM/CSIC), Universidad Autónoma de Madrid, Cantoblanco, 28049 Madrid, Spain \\ ${ }^{3}$ Campus of International Excellence UAM + CSIC, Cantoblanco, 28049 Madrid, Spain \\ ${ }^{4}$ Instituto de Física de Cantabria (CSIC-UC), 39005 Santander, Spain
}

Received: 28 May 2020 / Accepted: 20 August 2020 / Published online: 24 September 2020

(C) The Author(s) 2020

\begin{abstract}
An important task at future colliders is the measurement of the triple Higgs coupling. Depending on its size relative to the Standard Model (SM) value, certain collider options result in a higher experimental accuracy. Within the framework of Two Higgs Doublet Models (2HDM) types I and II we investigate the allowed ranges for all triple Higgs couplings involving at least one light, SM-like Higgs boson. We take into account theoretical constraints (unitarity, stability), experimental constraints from direct Higgs-boson searches, measurements of the SM-like Higgs-boson properties, flavor observables and electroweak precision data. We find that the SM-type triple Higgs coupling w.r.t. its SM value, $\lambda_{h h h} / \lambda_{\mathrm{SM}}$, can range between $\sim-0.5$ and $\sim 1.5$. Depending on which value is realized, the HL-LHC can compete with, or is clearly inferior to the ILC. We find the coupling $\lambda_{h h H}$ between $\sim-1.5$ and $\sim 1.5$. Triple Higgs couplings involving two heavy Higgs bosons, $\lambda_{h H H}, \lambda_{h A A}$ and $\lambda_{h H^{+} H^{-}}$can reach values up to $\mathcal{O}(10)$, roughly independent of the 2HDM type. This can lead to potentially strongly enhanced production of two Higgs-bosons at the HL-LHC or high-energy $e^{+} e^{-}$colliders.
\end{abstract}

\section{Introduction}

In 2012 the ATLAS and CMS collaborations have discovered a new particle that - within theoretical and experimental uncertainties - is consistent with the existence of a StandardModel (SM) Higgs boson at a mass of $\sim 125 \mathrm{GeV}$ [1-3]. No conclusive signs of physics beyond the SM have been found so far at the LHC. However, the measurements of Higgsboson couplings, which are known experimentally to a precision of roughly $\sim 20 \%$, leave room for Beyond Standard-

\footnotetext{
a e-mail: Francisco.Arco@uam.es

b e-mail: Sven.Heinemeyer@cern.ch (corresponding author)

c e-mail: Maria.Herrero@uam.es
}

Model (BSM) interpretations. Many BSM models possess extended Higgs-boson sectors. Consequently, one of the main tasks of the LHC Run III and beyond is to determine whether the observed scalar boson forms part of the Higgs sector of an extended model.

A key element in the investigation of the Higgs-boson sector is the measurement of the trilinear Higgs coupling of the SM-like Higgs boson, $\lambda_{h h h}$ (for recent reviews on Higgs couplings measurements at future colliders see $[4,5])$. In the case of a BSM Higgs-boson sector, equally important is the measurement of BSM trilinear Higgs-boson couplings. Most experimental studies assume the SM value of $\lambda_{h h h}$. However, in BSM models this coupling may differ significantly from its $\mathrm{SM}$ value. The expected achievable precision at different future colliders in the measurement of $\lambda_{h h h}$ depends on the value realized in nature.

A natural extension of the Higgs-boson sector of the SM is the "Two Higgs Doublet Model" (2HDM) (for reviews see, e.g., [6-8]). This model contains five physical Higgs bosons: the light and the heavy $\mathcal{C P}$-even $h$ and $H$, the $\mathcal{C P}$ odd $A$, and the pair of charged Higgs bosons, $H^{ \pm}$. The mixing angle $\alpha(\beta)$ diagonalizes the $\mathcal{C P}$-even (-odd) Higgs bosons and $\tan \beta$ is given by the ratio of the two vacuum expectation values, $\tan \beta:=v_{2} / v_{1}$. We assume for this work that the light $\mathcal{C P}$-even Higgs-boson $h$ is SM-like with a mass of $m_{h} \sim 125 \mathrm{GeV}$. All other Higgs bosons are assumed to be heavier. To avoid flavor changing neutral currents at treelevel, a $Z_{2}$ symmetry is imposed [9], possibly softly broken by the parameter $m_{12}^{2}$. Depending on how this symmetry is extended to the fermion sector, four types of the 2HDM can be realized: types I and II, lepton specific and flipped [7]. In the 2HDM also the stability conditions for the Higgs potential change with respect to the SM [10] (for a review see [11]).

In this paper, focusing on the 2HDM type I and II, we investigate the allowed ranges for all triple Higgs couplings involving at least one light, SM-like Higgs boson. Specifi- 
cally: $\lambda_{h h h}, \lambda_{h h H}, \lambda_{h H H}, \lambda_{h A A}$ and $\lambda_{h H^{+} H^{-}}$. The allowed ranges are obtained taking into account: theoretical constraints from unitarity and stability (we use [11-13]), experimental constraints from direct Higgs-boson searches (we use HiggsBounds [14-17], with data from [18-26]), the experimental production and decay rates of the SM-like Higgs boson (we use HiggsSignals [27,28], where the experimental data is listed in [29]), flavor observables (we use SuperIso [30,31], complemented with [32-34] and data from [35-45]) and electroweak precision observables (EWPO) (we use $S, T$ and $U[46,47]$, complemented with $[48,49]$ and bounds from [45]). Besides the allowed ranges, in this work we also present a detailed study of the dependence of the triple Higgs couplings on the free parameters of the model (to explore the 2HDM parameter space we use $2 \mathrm{HDMC}[50])$. The main interest in the allowed ranges for the triple Higgs couplings is that they may affect the rates of multiple Higgs boson production at current and future colliders. In particular, the production of Higgs boson pairs like $h h$, $h H, H H, h A, h H^{ \pm}, A A$ and $H^{+} H^{-}$could be significantly affected by the presence of sizable triple Higgs couplings within the 2HDM, yet allowed by the present constraints.

One of the key points of our study when exploring the parameter space of the 2HDM type I and II under the given constraints is the following: the primary focus of our explorations was to find allowed parameters that lead to either large non-SM triple Higgs boson couplings, or to large deviations from unity in the ratio of the light triple Higgs-boson coupling w.r.t. its SM value, $\lambda_{h h h} / \lambda_{\mathrm{SM}}$. In particular, we have explored scenarios with relatively heavy masses $m_{H}$, $m_{A}$ and $m_{H^{ \pm}}$near $1 \mathrm{TeV}$, but not enforcing the so-called alignment limit, $\cos (\beta-\alpha) \rightarrow 0$ (see, e.g., [51]). Furthermore, we have investigated the dependences of the allowed triple Higgs couplings on the soft $Z_{2}$-breaking parameter $m_{12}^{2}$. As we will see, $m_{12}^{2}$ plays a very important role in our search of sizable triple Higgs couplings. Finding a way to obtain large values for $m_{12}^{2}$, still being allowed by experimental data and by the theoretical constraints, turned out to be crucial in the course of this work. This also constitutes one of the main differences between our present study and previous studies on constraints in the $2 \mathrm{HDM}$, from LHC physics [52-54], EWPO [48,55,56], flavor physics [57] and global fits [51,58-60]. The relevance of $m_{12}^{2}$ in the context of large triple Higgs couplings in the 2HDM type II was also studied in $[61,62]$ (with the then available data). Furthermore, in this paper, we also explore special choices for $m_{12}^{2}$ in relation with other $2 \mathrm{HDM}$ parameters, like $m_{H}, \tan \beta$ and $\cos (\beta-\alpha)$. In particular, we explore the implications of the setting $m_{12}^{2}=m_{H}^{2} \cos ^{2} \alpha / \tan \beta$ (as considered previously, e.g., in [63]).

Our paper is organized as follows. In Sect. 2 we discuss details of the $2 \mathrm{HDM}$ and fix our notation. The experimental expectations for the measurement of $\lambda_{h h h}$ are briefly
Table 1 Allowed fermion couplings in the four types of 2HDM

\begin{tabular}{llll}
\hline & $u$-type & $d$-type & Leptons \\
\hline Type I & $\Phi_{2}$ & $\Phi_{2}$ & $\Phi_{2}$ \\
Type II & $\Phi_{2}$ & $\Phi_{1}$ & $\Phi_{1}$ \\
Type III (lepton-specific) & $\Phi_{2}$ & $\Phi_{2}$ & $\Phi_{1}$ \\
Type IV (flipped) & $\Phi_{2}$ & $\Phi_{1}$ & $\Phi_{2}$ \\
\hline
\end{tabular}

reviewed in Sect. 3. We discuss in Sect. 4 the theoretical and experimental constraints applied to our sampling of the 2HDMs. The numerical results are presented in Sect. 5. Here we show the maximum deviations of $\lambda_{h h h}$ from the SM that are still allowed taking into account all constraints. We also present the values that can be reached for the other triple Higgs couplings involving at least one $h$. Our conclusions are given in Sect. 6.

\section{The two Higgs doublet model}

We assume the $\mathcal{C P}$ conserving 2HDM. The scalar potential of this model can be written as [8]:

$$
\begin{aligned}
V= & m_{11}^{2}\left(\Phi_{1}^{\dagger} \Phi_{1}\right)+m_{22}^{2}\left(\Phi_{2}^{\dagger} \Phi_{2}\right)-m_{12}^{2}\left(\Phi_{1}^{\dagger} \Phi_{2}+\Phi_{2}^{\dagger} \Phi_{1}\right) \\
& +\frac{\lambda_{1}}{2}\left(\Phi_{1}^{\dagger} \Phi_{1}\right)^{2}+\frac{\lambda_{2}}{2}\left(\Phi_{2}^{\dagger} \Phi_{2}\right)^{2} \\
& +\lambda_{3}\left(\Phi_{1}^{\dagger} \Phi_{1}\right)\left(\Phi_{2}^{\dagger} \Phi_{2}\right)+\lambda_{4}\left(\Phi_{1}^{\dagger} \Phi_{2}\right)\left(\Phi_{2}^{\dagger} \Phi_{1}\right) \\
& +\frac{\lambda_{5}}{2}\left[\left(\Phi_{1}^{\dagger} \Phi_{2}\right)^{2}+\left(\Phi_{2}^{\dagger} \Phi_{1}\right)^{2}\right],
\end{aligned}
$$

where $\Phi_{1}$ and $\Phi_{2}$ denote the two $S U(2)_{L}$ doublets. To avoid the occurrence of tree-level flavor changing neutral currents (FCNC), a $Z_{2}$ symmetry is imposed on the scalar potential of the model under which the scalar fields transform as:

$\Phi_{1} \rightarrow \Phi_{1}, \quad \Phi_{2} \rightarrow-\Phi_{2}$

This $Z_{2}$, however, is softly broken by the $m_{12}^{2}$ term in the Lagrangian. The extension of the $Z_{2}$ symmetry to the Yukawa sector forbids tree-level FCNCs. This results in four variants of $2 \mathrm{HDM}$, depending on the $Z_{2}$ parities of the fermions. Table 1 lists the couplings for each type of fermion allowed by the $Z_{2}$ parity in four different types of $2 \mathrm{HDM}$.

Taking the electroweak symmetry breaking (EWSB) minima to be neutral and $\mathcal{C P}$-conserving, the scalar fields after EWSB can be parameterized as: ${ }^{1}$

\footnotetext{
${ }^{1}$ We follow here the notation for the field components and field rotations as in [34].
} 
$\Phi_{1}=\left(\begin{array}{c}\phi_{1}^{+} \\ \frac{1}{\sqrt{2}}\left(v_{1}+\rho_{1}+i \eta_{1}\right)\end{array}\right), \quad \Phi_{2}=\left(\begin{array}{c}\phi_{2}^{+} \\ \frac{1}{\sqrt{2}}\left(v_{2}+\rho_{2}+i \eta_{2}\right)\end{array}\right)$,

where $v_{1}, v_{2}$ are the real vevs acquired by the fields $\Phi_{1}, \Phi_{2}$, respectively, with $\tan \beta:=v_{2} / v_{1}$ and they satisfy the relation $v=\sqrt{\left(v_{1}^{2}+v_{2}^{2}\right)}$ where $v \simeq 246 \mathrm{GeV}$ is the $\mathrm{SM}$ vev. The eight degrees of freedom above, $\phi_{1,2}^{ \pm}, \rho_{1,2}$ and $\eta_{1,2}$, give rise to three Goldstone bosons, $G^{ \pm}$and $G^{0}$, and five massive physical scalar fields: two $\mathcal{C} \mathcal{P}$-even scalar fields, $h$ and $H$, one $\mathcal{C P}$-odd one, $A$, and one charged pair, $H^{ \pm}$. These are defined by:

$$
\begin{aligned}
\phi_{1}^{ \pm} & =\cos \beta G^{ \pm}-\sin \beta H^{ \pm}, \\
\phi_{2}^{ \pm} & =\sin \beta G^{ \pm}+\cos \beta H^{ \pm}, \\
\eta_{1} & =\cos \beta G^{0}-\sin \beta A, \\
\eta_{2} & =\sin \beta G^{0}+\cos \beta A, \\
\rho_{1} & =\cos \alpha H-\sin \alpha h, \\
\rho_{2} & =\sin \alpha H+\cos \alpha h,
\end{aligned}
$$

where the mixing angle diagonalizing the $\mathcal{C P}$-even sector is denoted as $\alpha$.

From Eq. (1), one can see that there are altogether 8 independent parameters in the model,

$m_{11}^{2}, \quad m_{22}^{2}, \quad m_{12}^{2}, \quad \lambda_{i,} \quad i=1,5$.

However, one can use the two minimization conditions of the potential at the vacuum to substitute the bilinears $m_{11}^{2}$ and $m_{22}^{2}$ for $v$ and $\tan \beta$ :

$m_{11}^{2}=m_{12}^{2} \tan \beta-\frac{v^{2}}{2}\left[\lambda_{1} \cos ^{2} \beta+\left(\lambda_{3}+\lambda_{4}+\lambda_{5}\right) \sin ^{2} \beta\right]$,

$m_{22}^{2}=m_{12}^{2} \cot \beta-\frac{v^{2}}{2}\left[\lambda_{2} \sin ^{2} \beta+\left(\lambda_{3}+\lambda_{4}+\lambda_{5}\right) \cos ^{2} \beta\right]$.

Furthermore, the couplings $\lambda_{i}$ in Eq. (1) can be replaced by the physical scalar masses and mixing angles:

$$
\begin{aligned}
v^{2} \lambda_{1} & =\frac{1}{\cos ^{2} \beta}\left(m_{h}^{2} \sin ^{2} \alpha+m_{H}^{2} \cos ^{2} \alpha-\bar{m}^{2} \sin ^{2} \beta\right), \\
v^{2} \lambda_{2} & =\frac{1}{\sin ^{2} \beta}\left(m_{h}^{2} \cos ^{2} \alpha+m_{H}^{2} \sin ^{2} \alpha-\bar{m}^{2} \cos ^{2} \beta\right) \\
v^{2} \lambda_{3} & =\frac{\sin 2 \alpha}{\sin 2 \beta}\left(m_{H}^{2}-m_{h}^{2}\right)+2 m_{H^{ \pm}}^{2}-\bar{m}^{2} \\
v^{2} \lambda_{4} & =m_{A}^{2}-2 m_{H^{ \pm}}^{2}+\bar{m}^{2} \\
v^{2} \lambda_{5} & =\bar{m}^{2}-m_{A}^{2}
\end{aligned}
$$

where $m_{h} \leq m_{H}$ denote the masses of the $\mathcal{C P}$-even Higgsbosons, $m_{A}, m_{H^{ \pm}}$denote the masses of the physical $\mathcal{C P}$ - odd and charged Higgs bosons respectively and, for later convenience, we have defined a new mass squared parameter $\bar{m}^{2}$, derived from $m_{12}^{2}$, given by:

$\bar{m}^{2}=\frac{m_{12}^{2}}{\sin \beta \cos \beta}$.

We will study the 2HDM in the physical basis, where the free parameters of the model, which we use as input, are chosen as:

$c_{\beta-\alpha}, \quad \tan \beta, \quad v, \quad m_{h}, \quad m_{H}, \quad m_{A}, \quad m_{H^{ \pm}}, \quad m_{12}^{2}$.

From now on we use sometimes the short-hand notation $s_{x}=$ $\sin (x), c_{x}=\cos (x)$. In our analysis we will identify the lightest $\mathcal{C} \mathcal{P}$-even Higgs boson, $h$, with the one observed at $\sim 125 \mathrm{GeV}$.

The couplings of the Higgs bosons to SM particles are modified w.r.t. the SM Higgs-coupling predictions due to the mixing in the Higgs sector. It is convenient to express the couplings of the neutral scalar mass eigenstates $h_{i}$ normalized to the corresponding SM couplings. We therefore introduce the coupling coefficients $c_{h_{i} V V}$ such that the couplings to the massive vector bosons are given by:

$$
\begin{aligned}
\left(g_{h_{i} W W}\right)_{\mu \nu} & =\mathrm{i} g_{\mu \nu}\left(c_{h_{i} V V}\right) g m_{W} \text { and } \\
\left(g_{h_{i} Z Z}\right)_{\mu \nu} & =\mathrm{i} g_{\mu \nu}\left(c_{h_{i} V V}\right) \frac{g m_{Z}}{c_{\mathrm{W}}},
\end{aligned}
$$

where $g$ is the $S U(2)_{L}$ gauge coupling, $c_{\mathrm{W}}$ the cosine of weak mixing angle, $c_{\mathrm{W}}=m_{W} / m_{Z}, s_{\mathrm{W}}=\sqrt{1-c_{\mathrm{W}}^{2}}$, and $m_{W}$ and $m_{Z}$ the masses of the $W$ boson and the $Z$ boson, respectively. For the $\mathcal{C} \mathcal{P}$-even boson couplings we have that $c_{h V V}=s_{\beta-\alpha}$ and $c_{H V V}=c_{\beta-\alpha}$ whereas the $\mathcal{C P}$-odd is $c_{A V V}=0$.

In the Yukawa sector, the discrete $Z_{2}$ symmetry leads to the following Lagrangian:

$$
\begin{aligned}
\mathcal{L}_{\mathrm{Yuk}}= & -\sum_{f=u, d, l} \frac{m_{f}}{v}\left[\xi_{h}^{f} \bar{f} f h+\xi_{H}^{f} \bar{f} f H+i \xi_{A}^{f} \bar{f} \gamma_{5} f A\right] \\
& -\left[\frac{\sqrt{2}}{v} \bar{u}\left(m_{u} V_{\mathrm{CKM}} \xi_{A}^{u} P_{L}+V_{\mathrm{CKM}} m_{d} \xi_{A}^{d} P_{R}\right) d H^{+}\right. \\
& \left.+\frac{\sqrt{2} m_{l}}{v} \xi_{A}^{l} \bar{v} P_{R} l H^{+}+\text {h.c. }\right],
\end{aligned}
$$

where the coefficients $\xi_{h_{i}}^{f}$ are defined in Table 2 for type I and II. The parameters $\xi_{h, H}^{f}$ can be interpreted as the ratio of the Higgs coupling with the fermions w.r.t. the SM coupling.

The potential of the $2 \mathrm{HDM}$ produces new interactions in the scalar sector. In this paper we will study in detail the couplings of the lightest $\mathcal{C} \mathcal{P}$-even Higgs boson with the other BSM bosons, concretely $\lambda_{h h h}, \lambda_{h h H}, \lambda_{h H H}, \lambda_{h A A}$ and 
Table 2 Yukawa couplings relative to the SM for $h$ (upper part), $H$ (middle part) and $A$ (lower part) in the 2HDM type I (II) in the middle (right) column

\begin{tabular}{lll}
\hline & Type I & Type II \\
\hline$\xi_{h}^{u}$ & $s_{\beta-\alpha}+c_{\beta-\alpha} \cot \beta$ & $s_{\beta-\alpha}+c_{\beta-\alpha} \cot \beta$ \\
$\xi_{h}^{d, l}$ & $s_{\beta-\alpha}+c_{\beta-\alpha} \cot \beta$ & $s_{\beta-\alpha}-c_{\beta-\alpha} \tan \beta$ \\
$\xi_{H}^{u}$ & $c_{\beta-\alpha}-s_{\beta-\alpha} \tan \beta$ & $c_{\beta-\alpha}-s_{\beta-\alpha} \tan \beta$ \\
$\xi_{H}^{d, l}$ & $c_{\beta-\alpha}-s_{\beta-\alpha} \tan \beta$ & $c_{\beta-\alpha}+s_{\beta-\alpha} \tan \beta$ \\
$\xi_{A}^{u}$ & $-\cot \beta$ & $-\cot \beta$ \\
$\xi_{A}^{d, l}$ & $\cot \beta$ & $-\tan \beta$ \\
\hline
\end{tabular}

$\lambda_{h H^{+} H^{-}}$. We define these $\lambda_{h h_{i} h_{j}}$ couplings such that the Feynman rules are given by:

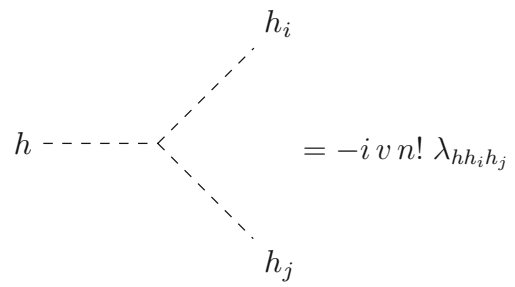

where $n$ is the number of identical particles in the vertex. The explicit expressions for the couplings $\lambda_{h h_{i} h_{j}}$ are shown in "Appendix A". We adopt this notation so the light Higgs trilinear has the same definition as in the SM, i.e. $-6 i v \lambda_{\mathrm{SM}}$ with $\lambda_{\mathrm{SM}}=m_{h}^{2} / 2 v^{2} \simeq 0.13$.

It should be noted that all the couplings of the $\mathcal{C P}$-even Higgs bosons strongly depend on $c_{\beta-\alpha}$. In particular, if $c_{\beta-\alpha}=0$ one can recover all the interactions of the SM Higgs boson for the $h$ state, what is known as the alignment limit. This limit is very interesting because, as we will discuss in Sect. 4.3, the Higgs measurements in colliders seem to overall agree with the SM values. However, in the alignment limit in general one can still have BSM physics related to the Higgs sector, like $h H^{+} H^{-}$or $Z H A$ interactions for example. On the other hand, the parameter $m_{12}^{2}$ may have a relevant impact on the triple Higgs boson couplings. In the alignment limit, it does not affect the couplings $\lambda_{h h h}$ and $\lambda_{h h H}$, but there are potentially relevant effects on the other couplings, $\lambda_{h H H}, \lambda_{h A A}$ and $\lambda_{h H^{+} H^{-}}$. Outside the alignment limit (i.e. for $\left|s_{\beta-\alpha}\right| \lesssim 1$ ) the effect of $m_{12}^{2}$ can also enter in a relevant way into $\lambda_{h h h}$ and $\lambda_{h h H}$.

\section{Experimental expectations for $\lambda_{h h h}$}

A determination of $\lambda_{h h h}$ (at different degrees of precision) will be able at future collider experiments. Various production cross sections show different dependences on $\lambda_{h h h}$, making those channels complementary to each other. Most evalu- ations of the anticipated experimental precision in $\lambda_{h h h}$ focus on the SM value. However, as we will analyze below, substantially different values of $\lambda_{h h h}$ are possible in the $2 \mathrm{HDM}$ (and other BSM models). The potential for the measurement of $\lambda_{h h h}$ at a future collider experiment thus strongly depends on the value of $\kappa_{\lambda}:=\lambda_{h h h} / \lambda_{\text {SM }}$ that is realized in nature.

In Fig. 1 we show the the various double Higgs production cross sections in the SM in $p p$ collisions with $\sqrt{s}=14 \mathrm{TeV}$ at (next-to) leading order ((N)LO) QCD, see Ref. [64] for details. The largest cross section is given by $g g \rightarrow h h{ }^{2}$ which will be most relevant for the measurement of $\lambda_{h h h}$ at the HL-LHC. One can see that the production cross section has a minimum around $\kappa_{\lambda} \sim 2$. Consequently, if such a value was realized, it is expected (see below) that the future experimental precision would be worse than for, e.g., $\kappa_{\lambda}=1$, where a determination at the level of $\sim 50 \%$ is anticipated [65]. Largest production cross sections, on the other hand, are for negative $\kappa_{\lambda}$. Consequently, a BSM model with very small or even negative values of $\kappa_{\lambda}$ is expected to result in a better determination of $\lambda_{h h h}$. A similar behavior is observed for the second largest production channel, the WBF channel $p p \rightarrow h h \mathrm{jj}$ (where $\mathrm{j}$ denotes a jet), with a minimum around $\kappa_{\lambda} \sim 1.5$. Different dependences are observed for the other, less relevant channels.

Similarly, in Fig. 2 we show the dependence on $\delta \kappa_{\lambda}:=$ $\kappa_{\lambda}-1$ for the Higgs-strahlung process, $e^{+} e^{-} \rightarrow Z h h$ (left) and the weak-boson fusion (WBF) channel, $e^{+} e^{-} \rightarrow v \bar{v} h h$ (right) for various center-of-mass energies, $\sqrt{s}$, at the ILC and CLIC [66]. Also indicated as horizontal colored bands are the anticipated experimental accuracies at the ILC500 (left) and ILC 1TeV, CLIC 1.4TeV and CLIC 3TeV (right). As for the HL-LHC, also at $e^{+} e^{-}$colliders the different production channels exhibit a different dependence on $\lambda_{h h h}$. For the Higgs-strahlung process smaller (larger) cross sections are obtained for smaller (larger) $\kappa_{\lambda}$. Higher values of $\sqrt{s}$ yield a weaker dependence on $\lambda_{h h h}$, as well as a smaller absolute cross section (as typical for $s$-channel processes). Consequently, a determination of $\lambda_{h h h}$ based (only) on the Higgs-strahlung channel is expected to be best at lower $\sqrt{s}$ (e.g. at the ILC500) and for larger values of $\kappa_{\lambda}$. The WBF channel exhibits a minimum at $\delta \kappa_{\lambda} \sim 0.5$. As for the Higgsstrahlung channel the dependence becomes weaker for larger values of $\sqrt{s}$, whereas the absolute values of the cross section increase with $\sqrt{s}$ (as typical for $t$-channel processes). Consequently, a case-by-case study is necessary to take into account the different, opposing effects.

The results of such a case-by-case study are shown in Fig. $3[67,68]$. Depicted are the relative (left) and absolute (right) accuracies of a determination of $\lambda_{h h h}$ (" $\lambda_{\text {meas }} / \lambda_{\text {true }}$ ") as a function of $\kappa_{\lambda}$ (" $\lambda_{\text {true }} / \lambda_{\mathrm{SM}}$ ") in the range of $-0.5 \ldots 2$.

\footnotetext{
${ }^{2}$ We denote here the Higgs boson at $\sim 125 \mathrm{GeV}$ with $h$. In Fig. 1, taken from Ref. [64], it is denoted as $H$.
} 
Fig. 1 Production cross sections for a pair of SM Higgs bosons as a function of $\lambda_{h h h} / \lambda_{\text {SM }}$ at the LHC [64]

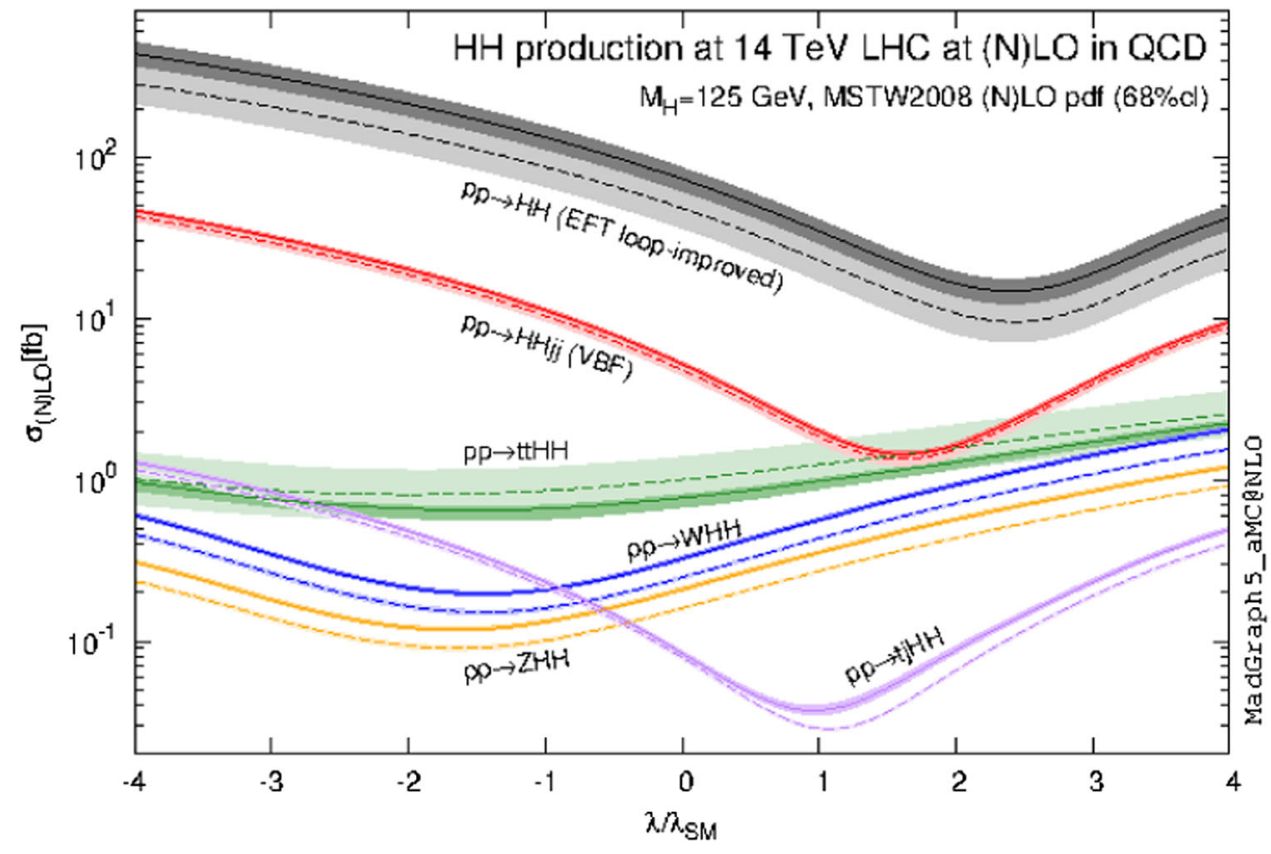

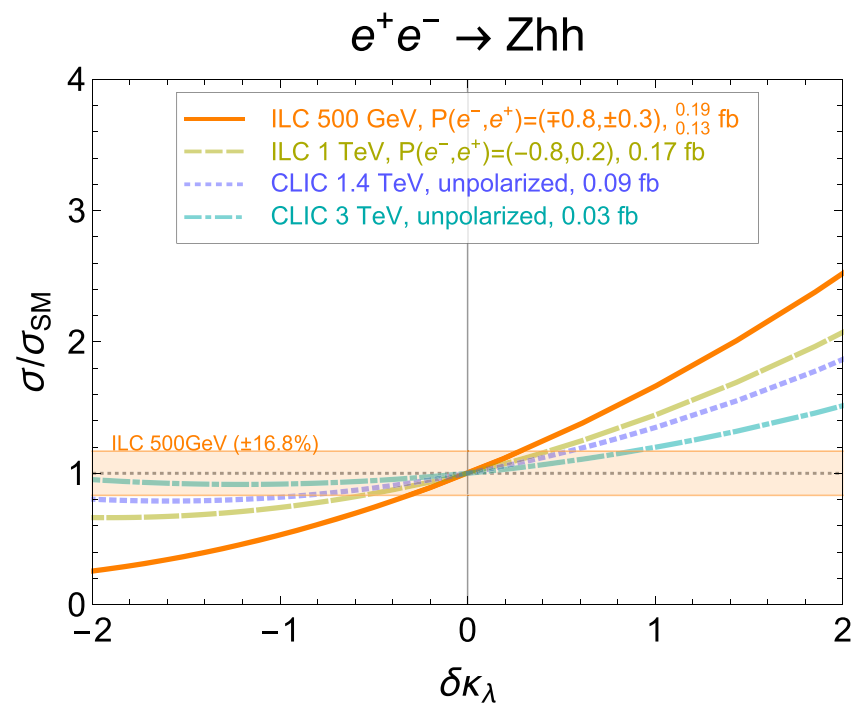

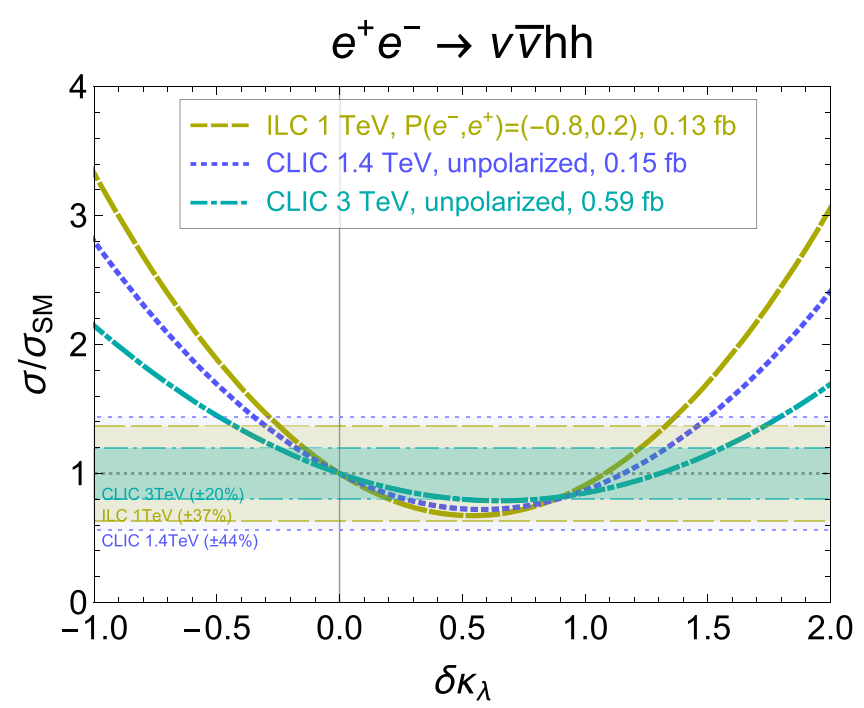

Fig. 2 Higgs-strahlung (left) and WBF production (right) of a pair of SM Higgs bosons as a function of $\lambda_{h h h}$ at the ILC and CLIC [66]. It should be noted that the experimental precision on the total cross section indicated by the horizontal bands is valid only for the SM case

Compared are the anticipated HL-LHC precision (based on a scaling of the results for $\kappa_{\lambda}=1$ ), the ILC500 precision (i.e. using only the Higgs-strahlung channel) and the ILC500+ $1 \mathrm{TeV}$ accuracy (i.e. also using the WBF channel results). Here it should be kept in mind that the HL-LHC analysis assumes that the other Higgs-boson couplings take their SM value, whereas for the ILC analysis it has been shown that the inclusion of the variation of the other Higgsboson couplings does not lead to a degradation of the anticipated precision. It is worth mentioning that in all these analyses, other possible channels that might contribute to double
Higgs production in extensions of the SM, like for instance the 2HDM, are not considered.

The achievable precisions follow the cross section dependences discussed above. At the HL-LHC the most (im)precise determination is expected for smaller (larger) values of $\kappa_{\lambda}$. $\mathrm{A} \sim 35(70) \%$ relative precision is anticipated for $\kappa_{\lambda}=$ $-0.5(2.0)$, as can be seen in the left plot of Fig. 3. Using only ILC500 results better (worse) experimental determinations are expected for larger (smaller) values of $\kappa_{\lambda}$, ranging from $\sim 65 \%$ at $\kappa_{\lambda}=-0.5$ to $\sim 15 \%$ at $\kappa_{\lambda}=2.0$. The large relative uncertainties close to $\kappa_{\lambda}=0$ are caused exactly by 
the smallness of the triple Higgs coupling. As can be seen in the right plot of Fig. 3, the absolute determination of $\kappa_{\lambda}$ continuously improves with smaller $\kappa_{\lambda}$. The combination with the WBF measurements at $\sqrt{s}=1 \mathrm{TeV}$ yields a substantially better determination for all values of $\kappa_{\lambda}$, but no monotonous behavior is found, owing to the different opposing effects, as discussed above. Future precisions between $\sim 5 \%$ and $\sim 30 \%$ are expected, depending on the value of $\kappa_{\lambda}$ realized in nature. Again the largest relative uncertainties of up to $30 \%$ are found close to $\kappa_{\lambda}=0$, whereas the absolute determination exhibits a nearly constant very precise determination of $\kappa_{\lambda}$ in the interval $[-0.5,1.0]$.

These results clearly show that the physics potential of a future collider experiment strongly depends on the actual value of $\lambda_{h h h}$ realized in a BSM model. This motivates the analysis presented in the following sections showing which values of $\lambda_{h h h}$ (and other triple Higgs couplings) can be realized in 2HDMs, taking into account all existing experimental and theoretical constraints.

\section{Experimental and theoretical constraints}

In this section we will describe the various theoretical and experimental constraints considered in our scans.

\subsection{Constraints from electroweak precision data}

Constraints from the electroweak precision observables (EWPO) can, in a simple approximation, be expressed in terms of the oblique parameters $S, T$ and $U[46,47]$. This approximation holds if the BSM effects enter mainly via corrections to gauge boson self-energies, as it is the case for extended Higgs sectors. Under these assumptions, the corrections are independent of the Yukawa sector of the 2HDM, and therefore the same for all types.

In 2HDMs there is a strong correlation between $T$ and $U$, and it is known that $T$ is by far more constraining than $U$ [49]. Hence, $U$ can safely be dropped in the present analysis. Specifically, our criterion to accept a point in the 2HDM parameter space, as being in agreement with the EWPO data, is as follows. For a given choice of input parameters in Eq. (13) to be allowed by the experimental observation, we require that the prediction of the $S$ and the $T$ parameter are in agreement with their experimental values $S=0.02 \pm 0.10$ and $T=0.07 \pm 0.12$ [45]. In this section we will study and compare the requirement of agreement at the $1 \sigma$ and $2 \sigma$ level. In our posterior numerical analysis in Sect. 5 we will require agreement at $2 \sigma$.

In the $2 \mathrm{HDM}$, as mentioned above, the most constraining oblique parameter is $T$, thus, we will focus in the following of this section on the constraints from the $T$ parameter. In the forthcoming analysis in Sect. 5 we have checked that once the allowed regions by $T$ are set, these are also allowed by $S$ and $U$, i.e. effectively it is sufficient to require agreement of $T$ with its experimental value. One peculiarity of the $T$ parameter in the $2 \mathrm{HDM}$ is that it depends on the relative mass squared differences of the scalar Higgs bosons. This can be seen in the explicit expression for the $T$ parameter in the $\mathcal{C P}$ conserving $2 \mathrm{HDM}$ that is given by [48]:

$$
\begin{aligned}
T= & \frac{g^{2}}{64 \pi^{2} m_{W}^{2}}\left\{F\left(m_{A}^{2}, m_{H^{ \pm}}^{2}\right)+s_{\beta-\alpha}^{2}\left[F\left(m_{H}^{2}, m_{H^{ \pm}}^{2}\right)\right.\right. \\
& \left.-F\left(m_{H}^{2}, m_{A}^{2}\right)\right] \\
& +c_{\beta-\alpha}^{2}\left[F\left(m_{H^{ \pm}}^{2}, m_{h}^{2}\right)-F\left(m_{A}^{2}, m_{h}^{2}\right)\right] \\
& +3 s_{\beta-\alpha}^{2}\left[F\left(m_{H}^{2}, m_{Z}^{2}\right)-F\left(m_{H}^{2}, m_{W}^{2}\right)\right] \\
& +3 c_{\beta-\alpha}^{2}\left[F\left(m_{h}^{2}, m_{Z}^{2}\right)-F\left(m_{h}^{2}, m_{W}^{2}\right)\right] \\
& \left.-3\left[F\left(m_{h_{\mathrm{SM}}}^{2}, m_{Z}^{2}\right)-F\left(m_{h_{\mathrm{SM}}}^{2}, m_{W}^{2}\right)\right]\right\},
\end{aligned}
$$

where $F(x, y)=\frac{x+y}{2}-\frac{x y}{x+y} \log \frac{x}{y}$, and it satisfies that $F(x, x)=0$. Therefore, the contributions to $T$ become small when either the mass of $H$ or $A$ is sufficiently close to the mass of the charged Higgs boson $H^{ \pm}[55,56]$. This motivates us to define three different simplified scenarios to explore the parameter space that is allowed by the EWPO in the 2HDM: scenario A, where $m_{A}=m_{H^{ \pm}}$; scenario B, where $m_{H}=m_{H^{ \pm}}$and scenario $\mathrm{C}$ where the masses of all the BSM Higgs bosons are equal, $m_{H}=m_{A}=m_{H^{ \pm}}$. One can see from Eq. (17) that in scenario A the main contributions to the $T$ parameter vanish for any value of $c_{\beta-\alpha}$, whereas in scenario B a contribution proportional to $c_{\beta-\alpha}^{2} F\left(m_{A}^{2}, m_{H^{ \pm}}^{2}\right)$ still survives, that will remain small close to the alignment limit.

Our study of the impact of these scenarios in the prediction for the $T$ parameter for different values of $c_{\beta-\alpha}$ and $m_{H^{ \pm}}$is summarized in Fig. 4. The 2HDM parameter space is explored with the 2 HDMC code [50]. For a given set of input parameters and a given Yukawa type of the 2HDM, the code computes as output the mass spectrum, decay widths and branching ratios of all the Higgs bosons. It furthermore calculates the $S, T$ and $U$ parameters and contribution to the anomalous magnetic moment of the muon, $(g-2)_{\mu}$. In Fig. 4 it can be seen that for scenario A any mass splitting between $m_{H}$ and $m_{A}=m_{H^{ \pm}}$is allowed inside the $2 \sigma$ region even far from the alignment limit. However, this is not the case for scenario B, where it can be seen that the prediction for $T$ is only inside the $2 \sigma$ region close to the alignment limit. If one goes to higher values of $c_{\beta-\alpha}$ (plots on the right) there are some values of $m_{A}$ that are disallowed, for example when $m_{H^{ \pm}}=650 \mathrm{GeV}$ and $c_{\beta-\alpha}=0.25$ the allowed region is $m_{A}-m_{H^{ \pm}}<350 \mathrm{GeV}$ (upper right plot). This effect becomes stronger for larger 

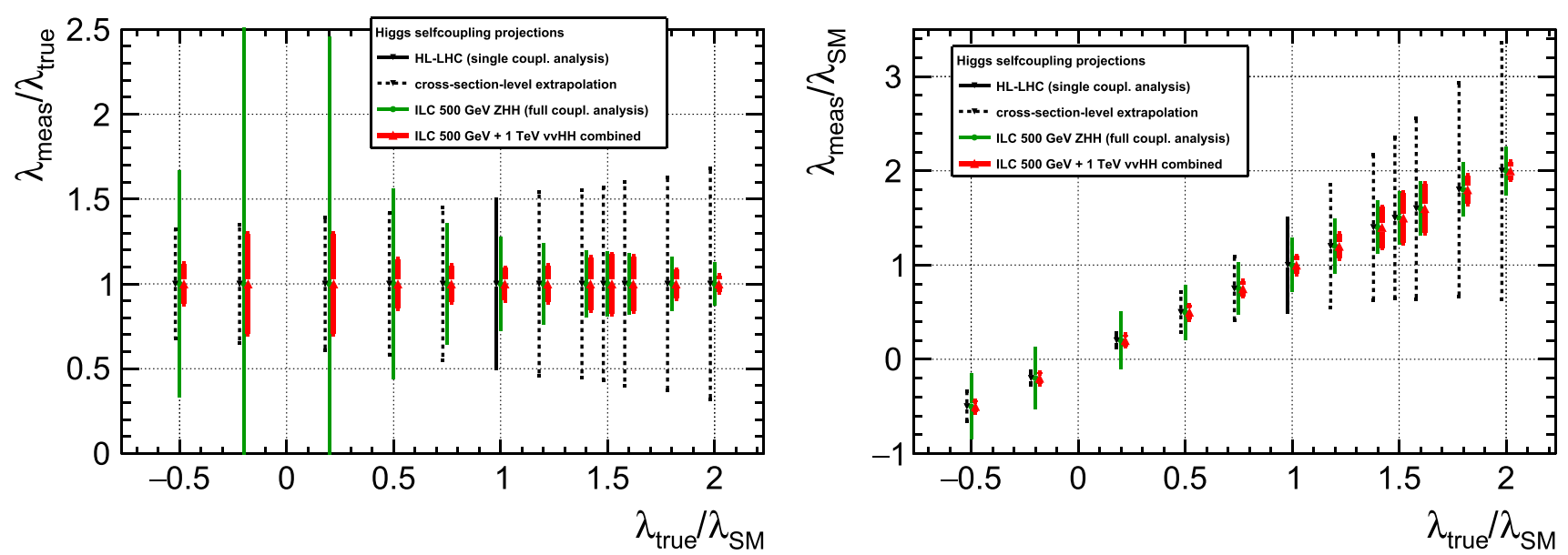

Fig. 3 Anticipated precision in the experimental determination of $\lambda_{h h h}$ as a function of $\lambda_{h h h} / \lambda_{h h h}^{\text {SM }}[67,68]$, relative (left) and absolute (right)

values of $m_{H^{ \pm}}$. For instance, for $m_{H^{ \pm}}=1000 \mathrm{GeV}$ and $c_{\beta-\alpha}=0.25$ (lower left plot) the allowed region shrinks to $-380 \mathrm{GeV}<m_{A}-m_{H^{ \pm}}<200 \mathrm{GeV}$. In general, scenario $\mathrm{A}$ and $\mathrm{C}$ (as a subset of scenario $\mathrm{A}$ ) is broadly allowed by $T$ for any value of $c_{\beta-\alpha}$ and mass splitting among the Higgs bosons, whereas scenario B can lead to a large deviation if $c_{\beta-\alpha}$ and $m_{H^{ \pm}}$increases (which is taken into account in Sect. 5 as discussed above).

\subsection{Theoretical constraints}

Like all models with extended scalar sectors, the 2HDM also faces important constraints coming from tree-level perturbartive unitarity and stability of the vacuum. We briefly describe these constraints below (for a discussion of higherorder effects and other considerations regarding the alignment limit, see, e.g., $[69,70])$.

\section{- Tree-level perturbative unitarity}

Perturbative unitarity is achieved by demanding that the eigenvalues of the lowest partial wave scattering matrices of the $2 \rightarrow 2$ processes in the scalar sector of the 2HDM, at the tree level, remain below $16 \pi$. This leads to the following constraints $[11,12]$ :

$$
\begin{aligned}
& \left|\lambda_{3} \pm \lambda_{4}\right| \leq 16 \pi \\
& \left|\lambda_{3} \pm \lambda_{5}\right| \leq 16 \pi \\
& \left|\lambda_{3}+2 \lambda_{4} \pm 3 \lambda_{5}\right| \leq 16 \pi \\
& \left|\frac{1}{2}\left(\lambda_{1}+\lambda_{2} \pm \sqrt{\left(\lambda_{1}-\lambda_{2}\right)^{2}+4 \lambda_{4}^{2}}\right)\right| \leq 16 \pi \\
& \left|\frac{1}{2}\left(\lambda_{1}+\lambda_{2} \pm \sqrt{\left(\lambda_{1}-\lambda_{2}\right)^{2}+4 \lambda_{5}^{2}}\right)\right| \leq 16 \pi \\
& \left|\frac{1}{2}\left(3 \lambda_{1}+3 \lambda_{2} \pm \sqrt{9\left(\lambda_{1}-\lambda_{2}\right)^{2}+4\left(2 \lambda_{3}+\lambda_{4}\right)^{2}}\right)\right| \leq 16 \pi
\end{aligned}
$$

It should be noted that the above requirement of tree level perturbative unitarity, limiting the maximum size of the given combinations of $\lambda_{i}$ 's, also ensures indirectly that the potential remains perturbative up to very high scales. Hence, in the present paper we do not incorporate additional constraints from other alternative criteria to require perturbativity that are based on limiting the size of the separate $\lambda_{i}$ 's which could be a priori more restrictive than the one applied here.

\section{- Stability}

First, we require the boundedness from below criterion. This criterion demands that the potential does not go to minus infinity when the field values approach infinity. This is fulfilled if the following conditions are satisfied $[10,11,13]$ :

$$
\begin{aligned}
& \lambda_{1} \geq 0 \\
& \lambda_{2} \geq 0 \\
& \lambda_{3}+\sqrt{\lambda_{1} \lambda_{2}} \geq 0 \\
& \lambda_{3}+\lambda_{4}-\left|\lambda_{5}\right|+\sqrt{\lambda_{1} \lambda_{2}} \geq 0
\end{aligned}
$$

Besides those inequalities, we will also demand that the minimum of the theory is a global minimum of the potential that can be achieved if [13]

$$
m_{12}^{2}\left(m_{11}^{2}-m_{22}^{2} \sqrt{\frac{\lambda_{1}}{\lambda_{2}}}\right)\left(\tan \beta-\sqrt[4]{\frac{\lambda_{1}}{\lambda_{2}}}\right) \geq 0 .
$$

According to Eqs. (7)-(11) the size of the triple couplings $\lambda_{i}$ are closely related to the size of the masses of the Higgs bosons and $m_{12}^{2}$. In general, the size of the triple Higgs couplings involving one $h$ and two heavy Higgs bosons grow with the corresponding heavy Higgs mass and, therefore, they can be large for large heavy masses, near the TeV scale. Consequently, unitarity sets limits on the maximum allowed 

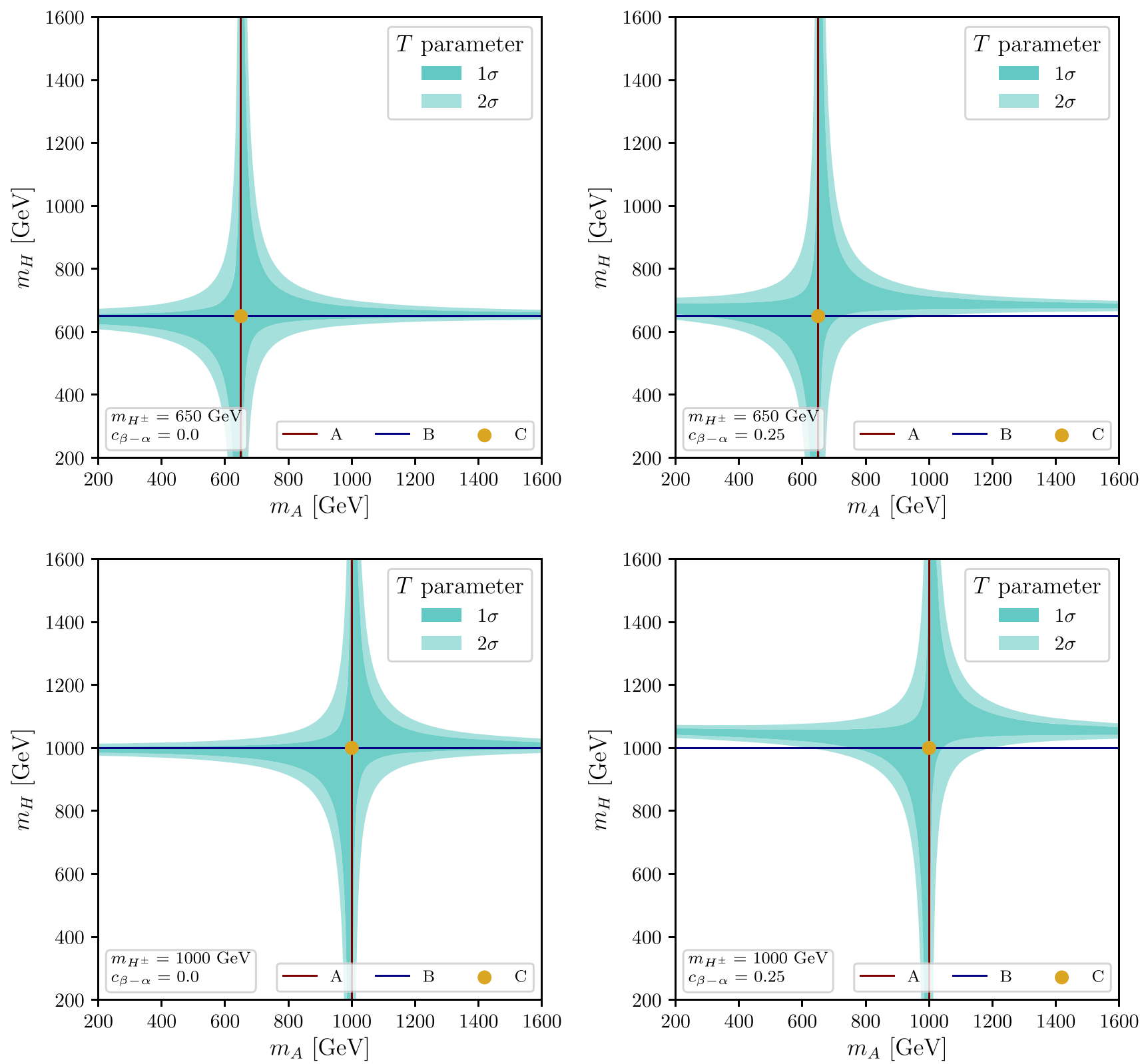

Fig. $4 T$ parameter for $m_{H^{ \pm}}=650 \mathrm{GeV}$ (top) and $m_{H^{ \pm}}=1000 \mathrm{GeV}$ (bottom) in the alignment limit, $c_{\beta-\alpha}=0$, (left) and for $c_{\beta-\alpha}=0.25$ (right). In scenario A $m_{H^{ \pm}}=m_{A}$ (red lines), in scenario B $m_{H^{ \pm}}=m_{H}$ (blue lines), and in scenario $\mathrm{C} m_{H^{ \pm}}=m_{H}=m_{A}$ (yellow points)

size of these large heavy masses, as can be seen in Fig. 5. One finds that only in scenarios where the heavy masses are large but nearly degenerate that these unitary bounds can be relaxed. The parameter $m_{12}^{2}$ also plays an important role in that concern. The plots on the right in Fig. 5 show that by setting the value of this parameter to $m_{12}^{2}=m_{H}^{2} \cos ^{2} \alpha / \tan \beta$, a diagonal corridor opens up allowing for larger values of these heavy masses above $1500 \mathrm{GeV}$ and with a considerable splitting. On the other hand, $m_{12}^{2}$ enters with a negative sign in some of the stability conditions (Eqs. (24)-(27)) and Eq. (28) imposes $m_{12}^{2} \geq 0$. Therefore if $m_{12}^{2}$ is large the Higgs boson masses should be also large to compensate those nega- tive contributions. In fact, setting $m_{12}^{2}$ to large values reduces considerably the allowed region by stability and shrinks it to the upper right corner in these two dimensional mass plots. This reduces as well the intersection area with the unitarity allowed region (dotted areas), as can be seen in the two plots in the middle with $m_{12}^{2}=100000 \mathrm{GeV}^{2}$. Here $\lambda_{1}$ plays an important role, as it contains a negative contribution $\propto m_{12}^{2}$ that grows with $\tan \beta$, see Eq. (7). This can drive $\lambda_{1}$ to negative values and yield disagreement with the stability condition in Eq. (24). One way to minimize this effect on $\lambda_{1}$ is to fix $m_{12}^{2}$ such that the two last terms in Eq. (7) cancel each other. This condition leads to the above commented equation allowing 

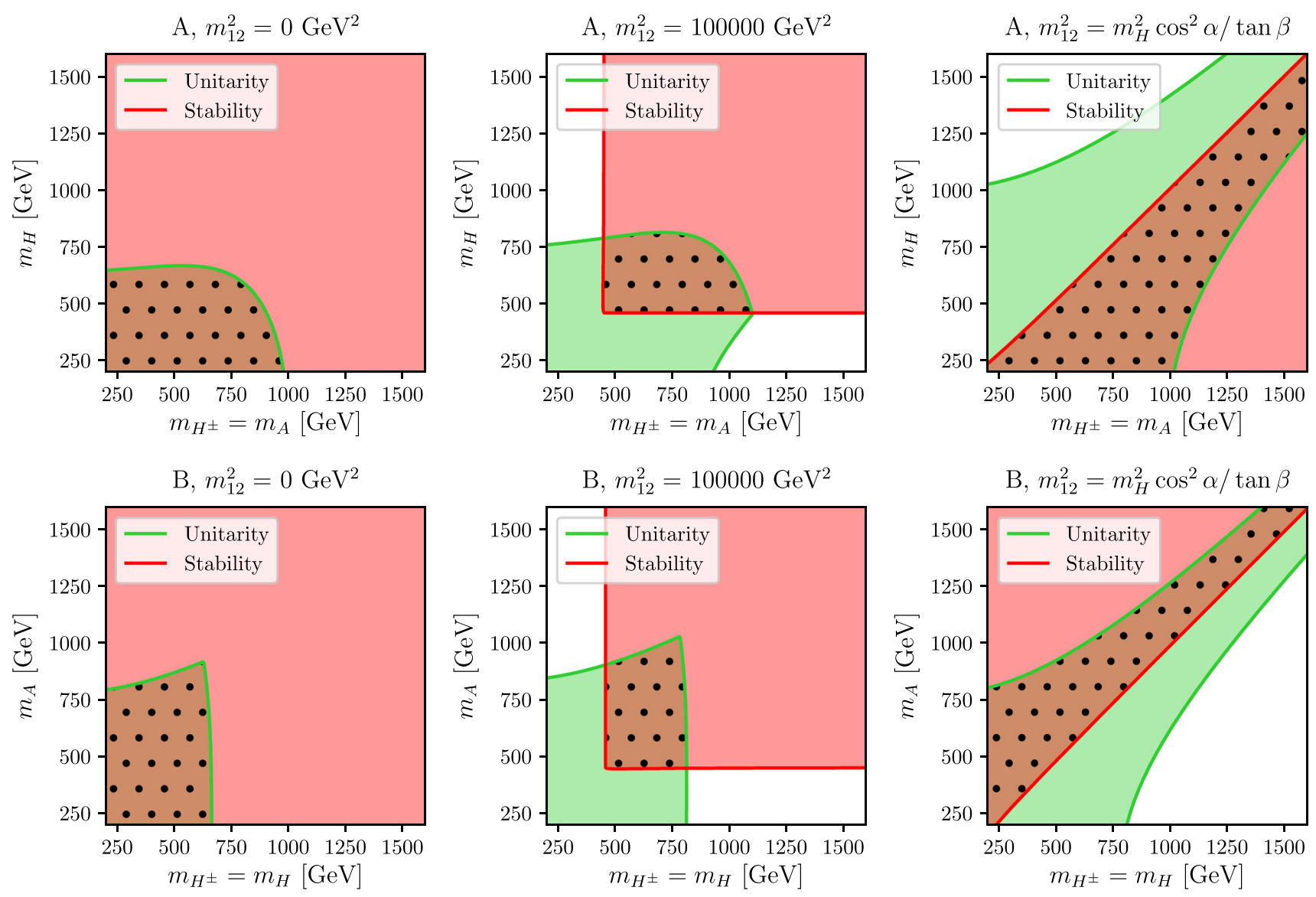

Fig. 5 Allowed areas in two selected Higgs masses of the 2HDM parameter space, delimited by the theoretical constraints from unitarity (green areas), stability (red areas), and both together (dotted areas), obtained from Eqs. (18) to (28). The alignment limit is assumed and $\tan \beta$ is fixed to $\tan \beta=1.5$ for scenario A (top plots) and scenario $\mathrm{B}$ (bottom plots). $m_{12}^{2}$ is set to 0 (left plots), $100000 \mathrm{GeV}^{2}$ (middle plots) and $m_{12}^{2}=m_{H}^{2} \cos ^{2} \alpha / \tan \beta$ (right plots) for the diagonal corridor in the right plots of Fig. 5 where the intersection region (dotted area) is clearly expanded. Therefore, to enlarge the allowed region by unitarity and stability in our forthcoming analysis we will consider this as a special interesting case where to explore the maximum allowed size of the triple Higgs couplings. This condition on $m_{12}^{2}$ has been considered previously [63] and can also be translated into a condition on $\bar{m}^{2}$, using Eq. (12),

$m_{12}^{2}=\frac{m_{H}^{2} \cos ^{2} \alpha}{\tan \beta}, \quad \bar{m}^{2}=\frac{m_{H}^{2} \cos ^{2} \alpha}{\sin ^{2} \beta}$

Regarding the comparison of the allowed regions for the two considered scenarios $\mathrm{A}$ and $\mathrm{B}$, we show in Fig. 5 some specific examples, for $\tan \beta=1.5$, where one can clearly see the impact of $m_{12}^{2} \neq 0$ and compare it with imposing Eq. (29). In the case when $m_{12}^{2}=0$ (left) all masses are allowed by stability but they are restricted by unitarity, and the final allowed dotted region is, in scenario A, for masses $m_{H^{ \pm}}=m_{A} \lesssim 1000 \mathrm{GeV}$ and $m_{H} \lesssim 650 \mathrm{GeV}$ and, in scenario $\mathrm{B}$, for masses roughly below $750 \mathrm{GeV}$. When $m_{12}^{2}$ increases (center) the allowed region by unitarity is similar to the previous situation, but due to the large value for $m_{12}^{2}$, now to get stability, the masses should be larger than approximately $500 \mathrm{GeV}$ in both scenarios $\mathrm{A}$ and $\mathrm{B}$. The situation is completely different in the right plots where Eq. (29) is adopted. In these cases masses can get very large values as well as $m_{12}^{2}$ and also splitting between the two free masses is allowed. This splitting stretches in both scenarios A and B as the masses grow and the final allowed region by stability and unitarity is confined to a diagonal corridor which is narrower in scenario B than in scenario A. It should be noted that in cases where Eq. (29) is satisfied, in order to cope with the theoretical constraints scenario A demands that $m_{H^{ \pm}}=m_{A} \geq m_{H}$ and scenario B that $m_{A} \geq m_{H^{ \pm}}=m_{H}$. The allowed region by both theoretical constraints in the left and center columns would dramatically shrink for a larger value of $\tan \beta$ because of the size of $\lambda_{1}$, but the right plots would remain similar. In some sense, Eq. (29) gives an upper limit for $m_{12}^{2}$ for large masses and large $\tan \beta$. 

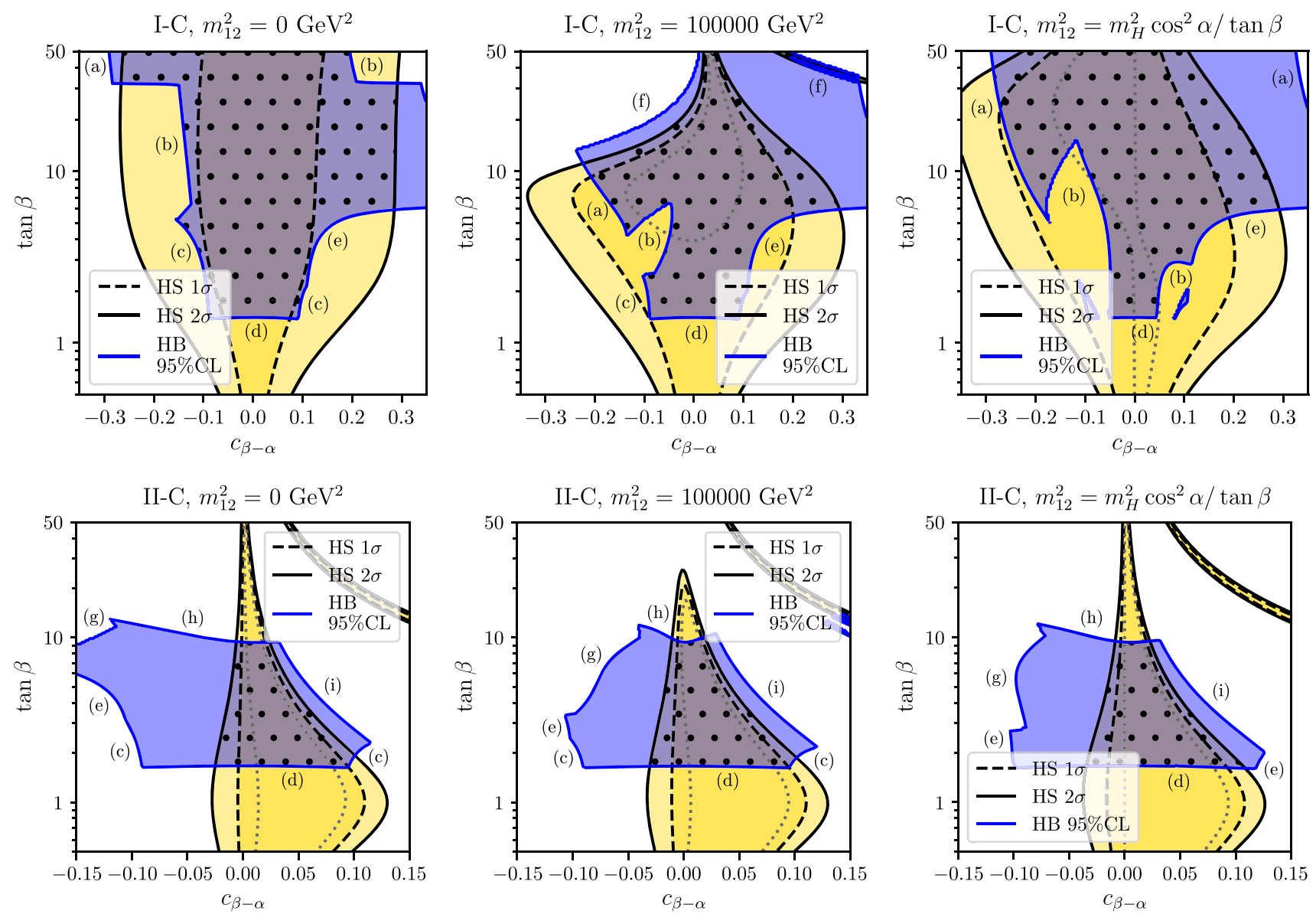

Fig. 6 Allowed regions of the $2 \mathrm{HDM}$ in the $\left(c_{\beta-\alpha}, \tan \beta\right)$ plane from BSM Higgs bosons searches and direct measurements of the $125 \mathrm{GeV}$ Higgs from HiggsBounds (blue regions) and Higgs Signals (yellow regions) in scenario $\mathrm{C}$ with $m_{H}=m_{A}=m_{H^{ \pm}}=650 \mathrm{GeV}$ for
Yukawa type I (top) and II (bottom) and different values of $m_{12}^{2}$. The dotted grey lines correspond to contours with the same $\chi^{2}$ for the $125 \mathrm{GeV}$ Higgs-boson rate measurements at the LHC as found in the SM

\subsection{Constraints from direct searches at colliders}

The $95 \%$ confidence level exclusion limits of all important searches for BSM Higgs bosons are included in the public code HiggsBounds V.5.3.2 [14-17], including Run 2 data from the LHC. Given a set of theoretical predictions in a particular model, HiggsBounds determines which is the most sensitive channel and determines, based on this most sensitive channel, whether the point is allowed or not at the 95\% CL. As input the code requires some specific predictions from the model, like branching ratios or Higgs couplings, that we computed with the help of the $2 \mathrm{HDMC}$ code (see Sect. 4.1). In Fig. 6 plotted in blue are shown the allowed regions of the $2 \mathrm{HDM}$ in the $\left(c_{\beta-\alpha}, \tan \beta\right)$ plane for the case where all the masses of the heavy Higgs bosons are set to $650 \mathrm{GeV}$, i.e. in the simplest scenario C. In the upper (lower) row we show the results for the 2HDM type I (II) with $m_{12}^{2}=$ 0, $100000 \mathrm{GeV}^{2}$ and set via Eq. (29) in the left, middle and right column, respectively. The particular exclusion channel that sets a bound limiting this blue region is specified with a Latin letter and corresponds to one of the following channels:

(a) $p p \rightarrow H \rightarrow h h \rightarrow(b \bar{b})\left(\tau^{+} \tau^{-}\right)$[18],

(b) $p p \rightarrow H \rightarrow h h \rightarrow(b \bar{b})\left(b \bar{b} / \tau^{+} \tau^{-} / W^{+} W^{-} / \gamma \gamma\right)$ [19],

(c) $p p \rightarrow H \rightarrow V V$ [20],

(d) $p p \rightarrow H^{ \pm} t b \rightarrow(t b) t b[21]$

(e) $g g \rightarrow A \rightarrow Z h \rightarrow\left(l^{+} l^{-}\right)(b \bar{b})$ [22],

(f) $p p \rightarrow h X \rightarrow \gamma \gamma X$ [23],

(g) $p p \rightarrow H \rightarrow h h \rightarrow(b \bar{b})(b \bar{b})$ [24],

(h) $p p \rightarrow H \rightarrow \tau^{+} \tau^{-}[25]$,

(i) $p p \rightarrow h \rightarrow Z Z \rightarrow\left(l^{+} l^{-}\right)\left(l^{+} l^{-}\right)$[26].

In broad terms, the 2HDM type I seems to be less constrained than type II by the searches of heavy Higgs bosons. Both types have a lower bound on $\tan \beta \sim 1.4$ from channel (d), whereas type II also has an upper bound given by channel (h). In type I negative values of $c_{\beta-\alpha}$ are constrained by channels (a), (b) and (c) while for positive values the more 
relevant channel is (e). On the other hand, in type II for a negative $c_{\beta-\alpha}$ channels (e) and (g) become the most restrictive ones, and in the positive $c_{\beta-\alpha}$ region channel (i) is the most sensitive one.

It is also worth to notice that for $m_{12}^{2}=100000 \mathrm{GeV}^{2}$ (center plots) there are more stringent bounds than in the other cases coming from channel (f) in type I and from channel ( $\mathrm{g}$ ) in type II. This is an example of how $m_{12}^{2}$ can be relevant in some situations when the contributions from the scalar sector are important. Clearly, the experimental bounds on BSM Higgs searches strongly depend on the masses of such particles, so the allowed contours and the exclusion channels shown in Fig. 6 will change for a different value of the masses. In general, for smaller values of the input masses the parameter space would be more constrained.

\subsection{Constraints from the SM-like Higgs-boson properties}

Any model beyond the SM has to accommodate the SM-like Higgs boson, with mass and signal strengths as they were measured at the LHC [1-3]. In our scans the compatibility of the $\mathcal{C P}$-even scalar $h$ with a mass of $125.09 \mathrm{GeV}$ with the measurements of signal strengths at Tevatron and LHC is checked with the code HiggsSignals V.2.2.3 [27, 28]. Higgs Signals provides a statistical $\chi^{2}$ analysis of the SM-like Higgs-boson predictions of a certain model compared to the measurement of Higgs-boson signal rates and masses from Tevatron and LHC. Again, the predictions of the 2HDM have been obtained with the 2HDMC code. The complete list of implemented experimental data can be found in Ref. [29]. Here and in our posterior analysis we will require that for a parameter point of the 2HDM to be allowed, the corresponding $\chi^{2}$ is within $2 \sigma\left(\Delta \chi^{2}=6.18\right)$ from the SM fit: $\chi_{\mathrm{SM}}^{2}=43.6$.

In Fig. 6 we present the results of HiggsSignals for scenario $\mathrm{C}$ with $m_{H}=m_{A}=m_{H^{ \pm}}=650 \mathrm{GeV}$ as a function of $\tan \beta$ and $c_{\beta-\alpha}$, which are the most relevant parameters to determine the couplings of the $h$ boson to the SM particles. In yellow are shown the allowed regions from HiggsSignals. (In blue are shown the allowed regions from HiggsBounds, as discussed in the previous section). In this figure we show the contours from HiggsSignals corresponding to a $1 \sigma$ (dashed lines) and $2 \sigma$ (solid lines) distance from the SM fit and the contours that have the same fit as the SM (dotted grey lines). In consequence, the regions inside these dotted grey lines have a better agreement with the experimental results that the SM. It can be seen that the parameter space is strongly constrained for $c_{\beta-\alpha}$ to be close to the alignment limit, such that $h$ behaves sufficiently SM-like. In particular, the $2 \sigma$ allowed region for the Yukawa type II (bottom) is substantially smaller compared to type I (top). In particular for type II, we find that negative values of $c_{\beta-\alpha}$ are very disfavored. The maximum deviation from the alignment limit takes place for $\tan \beta \sim 1$, where values between $c_{\beta-\alpha}=0.13$ and $c_{\beta-\alpha}=-0.03$ can be found inside the $2 \sigma$ region from the SM. However, as $\tan \beta$ increases the model is forced to be very close to the alignment limit to agree with the experimental data. This is caused by an enhancement of the coupling of $h$ to $b$-quark (see Table 2). It should be noted that in the type II fits a new allowed branch appears in the upper right part of the plot which corresponds to $\xi_{h}^{d}=-1$, known as the wrong sign Yukawa region. For type I the constraints are weaker, specially for $\tan \beta>3$, where we can accommodate inside the $2 \sigma$ region values for $c_{\beta-\alpha}$ up to \pm 0.3 . Fig. 6 also captures the role of $m_{12}^{2}$ in the fits. In type I $m_{12}^{2}$ barely changes the fits for $\tan \beta \lesssim 3$ region. However, the increment of $m_{12}^{2}$ narrows the $1 \sigma, 2 \sigma$ contours around the alignment limit, notably for $m_{12}^{2}=100000 \mathrm{GeV}^{2}$ (upper center) where the fit forces $c_{\beta-\alpha} \sim 0$ when $\tan \beta$ is large. In the case of type II the fits seems to be roughly independent of $m_{12}^{2}$, except again for $m_{12}^{2}=100000 \mathrm{GeV}^{2}$ where the model is completely outside the $2 \sigma$ region for $\tan \beta>25$.

In addition, it can be seen that an extensive region exists for both types that gives a better fit to the experimental data than the SM i.e. $\chi^{2}<\chi_{\mathrm{SM}}^{2}$, even though for type $\mathrm{I}_{12}^{2}$ is required to be different from zero. Such regions are expected due to the additional freedom in the 2HDM to accommodate the LHC measurements. For the sake of completeness, we would like to comment that the impact of $m_{H}, m_{A}$ and $m_{H^{ \pm}}$ could be important for the fit when they are low, because only then they could give sizable contributions to the light Higgs measurements, specially for the $H$ boson.

Other recent studies from $\mathrm{LHC}$ data analysis [52-54], also set similar constraints on the $\left(c_{\beta-\alpha}, \tan \beta\right)$ plane, since these are the most relevant 2HDM parameters (entering the Higgsboson couplings) at the LHC. One of the main differences to our study is that, as emphasized in the introduction, we have a strong focus on the role played by the $m_{12}^{2}$ parameter, which turns out to be relevant in our search of sizable triple Higgs couplings.

\subsection{Constraints from flavor physics}

Constraints from flavor physics have proven to be very significant in the 2HDM mainly because of the presence of the charged Higgs boson. Various flavor observables like rare $B$ decays, $B$ meson mixing parameters, $\operatorname{BR}\left(B \rightarrow X_{s} \gamma\right)$, LEP constraints on $Z$ decay partial widths etc., which are sensitive to charged Higgs boson exchange, provide effective constraints on the available parameter space $[57,58]$. Here we will take into account the decays $B \rightarrow X_{s} \gamma$ and $B_{s} \rightarrow \mu^{+} \mu^{-}$, which we find to be the most constraining 
ones and whose experimental values are (we use the average from [45]):

$$
\begin{aligned}
\mathrm{BR}\left(B \rightarrow X_{s} \gamma\right) & =(3.1 \pm 1.1) \times 10^{-4} \\
\mathrm{BR}\left(B_{s} \rightarrow \mu^{+} \mu^{-}\right) & =\left(2.7_{-0.5}^{+0.6}\right) \times 10^{-9}
\end{aligned}
$$

We will set our bounds in the $2 \sigma$ region from the central value according to the experimental value.

In order to compute the theoretical predictions in the 2HDM we have used the public code SuperIso [30,31] with the model input given by $2 \mathrm{HDMC}$. Moreover, we have included in SuperIso the contributions to the Wilson coefficient $C_{P}$ from the Higgs-penguin diagrams, that are missing in the public version and that can be relevant for the $\mathrm{BR}\left(B_{s} \rightarrow \mu^{+} \mu^{-}\right)$prediction [32-34].

In Fig. 7 we present the allowed regions from the flavor constraints in the $\left(m_{H^{ \pm}}, \tan \beta\right)$ plane in the alignment limit for scenario $\mathrm{C}$ (all masses of BSM bosons degenerated) for Yukawa types I (upper row) and II (lower row) for $m_{12}^{2}=0,100000 \mathrm{GeV}$ and set via Eq. (29) in the left, middle and right column, respectively. We show the regions allowed by $B \rightarrow X_{s} \gamma$ (pink areas) and by $B_{s} \rightarrow \mu^{+} \mu^{-}$(teal areas). Dotted areas are the intersections of these two allowed regions. The $2 \mathrm{HDM}$ contribution to the process $B \rightarrow X_{s} \gamma$ depends on the couplings of the $b$ and $s$ quarks with the other $u$-type quarks through a charged Higgs boson. As the Yukawa couplings of the charged Higgs bosons in the 2HDM scale like the ones of the $\mathcal{C P}$-odd Higgs boson, this coupling is given by a combination of $\xi_{A}^{u, d}$ (see Table 2) and the quark masses. In the case of model type I those couplings are enhanced for large values of $\cot \beta$, in consequence the region of low $\tan \beta$ is forbidden in the top plots of Fig. 7 and softly fades as the mass of the charged Higgs increases. On the contrary, in type II it is found a well known $\tan \beta$ independent constraint of $m_{H^{ \pm}}>500 \mathrm{GeV}$. The BSM contributions to $B \rightarrow X_{s} \gamma$ are induced from the Yukawa coupling and therefore neither $c_{\beta-\alpha}$ or $m_{12}^{2}$ affects the bounds, as it can be seen in the figure. Focusing on $B_{s} \rightarrow \mu^{+} \mu^{-}$one finds a similar constraint for low $\tan \beta$ on both model types due to analogous arguments discussed before for $B \rightarrow X_{s} \gamma$. Nevertheless, in model type II there is a disallowed region for large $\tan \beta$ and low masses. This is due to the contributions from the Higgs-penguin diagrams (mediated by $H$ and $h$ ) to the process $B_{s} \rightarrow \mu^{+} \mu^{-}$which are sensitive to $m_{12}^{2}$, via $\lambda_{H_{H} H^{-}}$ and $\lambda_{h H^{+} H^{-}}$from the loops involving charged Higgs bosons, and that are enhanced at large $\tan \beta$ (see also [33]). The largest effect from $m_{12}^{2}$ on $B_{S} \rightarrow \mu^{+} \mu^{-}$is from $\lambda_{H_{H} H^{-}}$since the $H$-penguin diagram goes as $\tan ^{3} \beta$, and this leads to relevant constraints in the large $\tan \beta$ and low $m_{H^{+}}$region. If, however, $m_{12}^{2}$ is fixed to Eq. (29) and if the alignment limit is taken, then the coupling $\lambda_{H_{H} H^{+}}$vanishes and in conse- quence the Higgs penguins contributions are not large enough to give a bound in that region.

\section{Numerical results}

In this section we analyze numerically which intervals (or extreme values) of the various triple Higgs boson couplings are still allowed, taking into account all experimental and theoretical constraints as discussed in Sect. 4. In the case of $\lambda_{h h h}$ this will give a guideline to which collider option may be needed to perform a precise experimental determination. For the triple Higgs couplings involving heavy Higgs bosons this will indicate in which processes large effects, e.g. possibly enhanced production cross sections, can be expected due to large triple Higgs couplings.

We perform our evaluation in both type I and type II models (and leave the other types for future investigations). We start our exploration with the "simplest" scenario C, but later also explore scenario A and B. In the headlines of our plots we indicate which type and which scenario are chosen. The other parameters are chosen such as to maximize either the deviations of $\lambda_{h h h}$ from it SM value (where the plots below show $\kappa_{\lambda}:=\lambda_{h h h} / \lambda_{\mathrm{SM}}$ ), or to maximize (positive or negative) the size of the triple Higgs couplings involving the heavy Higgs bosons (where the plots below show the triple Higgs couplings as defined in Eq. (16)).

\subsection{Scenario C}

We start with scenario C, i.e. $m_{H^{ \pm}}=m_{H}=m_{A},{ }^{3}$ and $m_{h}=125 \mathrm{GeV}$. In Figs. 8 and 9 we show the $\left(c_{\beta-\alpha}, \tan \beta\right)$ plane in the 2HDM type I, where $m_{12}^{2}$ is fixed by Eq. (29) to maximize the regions allowed by unitarity and stability of the potential, see Sect. 4.2. The common Higgs boson mass scale is set to $m_{H^{ \pm}}=1000 \mathrm{GeV}$. Dotted areas always refer to the intersections of the allowed regions by the various analysis involved. The first three panels of Fig. 8 indicate the restrictions from three sets of constraints. The upper left panel shows the areas allowed by HiggsBounds and HiggsSignals, as discussed in Sects. 4.3 and 4.4. One can see that a wide area roughly centered around $c_{\beta-\alpha}=0$ (i.e. the alignment limit) is allowed by the direct BSM Higgsboson searches as well as by the requirement that the Higgsboson at $\sim 125 \mathrm{GeV}$ is in agreement with the LHC rate measurements. The upper right plot shows the constraints from flavor physics, as discussed in Sect. 4.5. Following the explanations given there, in this realization of the type I scenario the two constraints result in lower limits on $\tan \beta$, where $B \rightarrow X_{s} \gamma$ gives the stronger constraint. The last set of constraints is given in the middle left plot, showing the effects of

\footnotetext{
${ }^{3}$ Here and in the following we will denote this common mass as $m_{H^{ \pm}}$.
} 

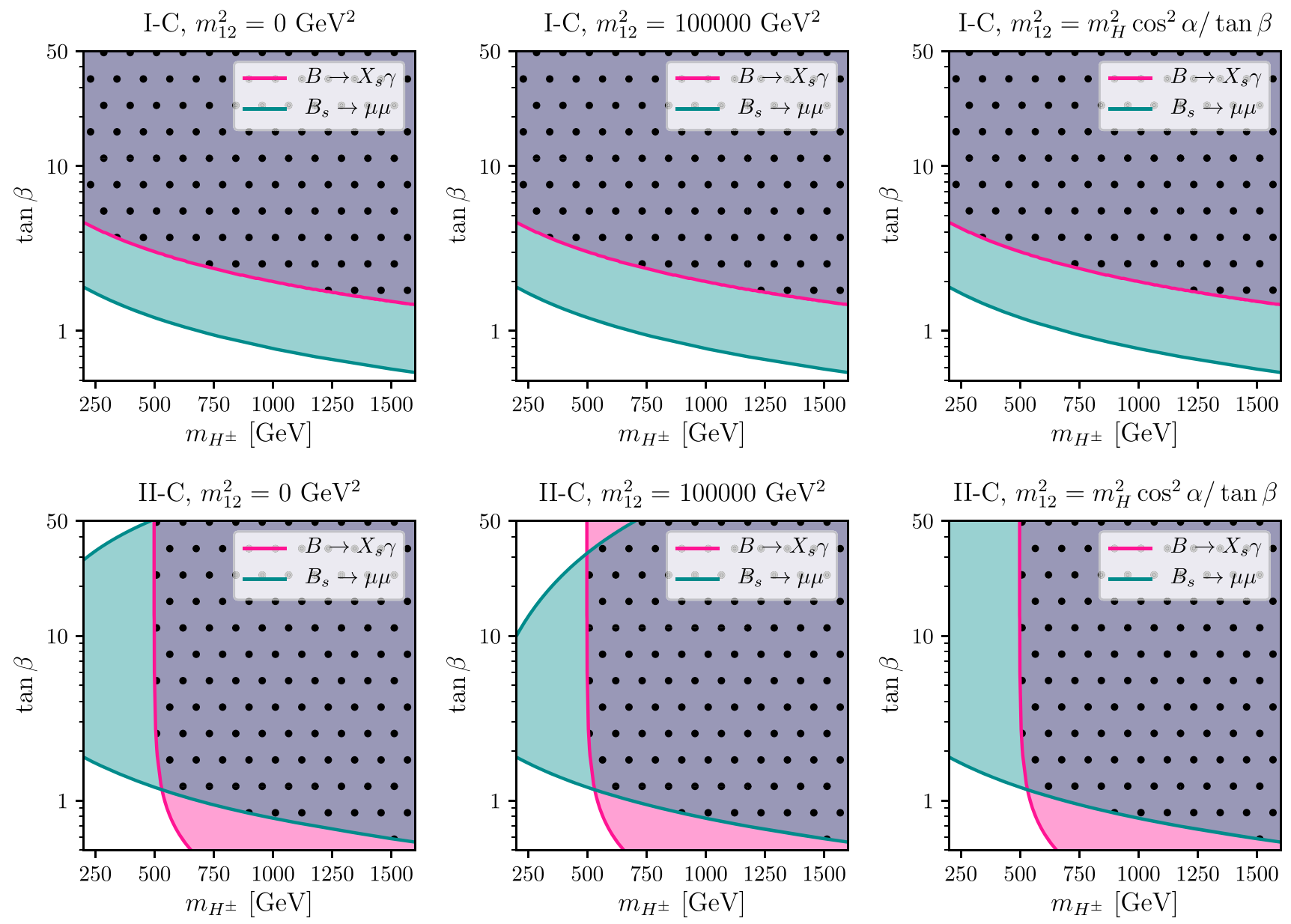

Fig. 7 Allowed regions in the $\left(m_{H^{ \pm}}, \tan \beta\right)$ plane of the 2HDM for scenario C with $m_{H}=m_{A}=m_{H^{ \pm}}$and for Yukawa types I (upper row) and II (lower row) for $m_{12}^{2}=0,100000 \mathrm{GeV}$ and set via Eq. (29) in the left, middle and right column, respectively. The alignment limit, $c_{\beta-\alpha}=0$, has been fixed. Pink areas are the allowed regions by $B \rightarrow X_{s} \gamma$ and teal areas those allowed by $B_{s} \rightarrow \mu^{+} \mu^{-}$. Dotted areas are the intersections of these two allowed regions requiring unitarity and stability of the potential as discussed in Sect. 4.2. The middle right plot indicates the intersection set of the three other panels. Being in scenario $C$ the electroweak precision constraints, see Sect. 4.1 are automatically fulfilled. In the $\left(c_{\beta-\alpha}, \tan \beta\right)$ plane this intersection defining the total allowed area starts at $\tan \beta \sim 2$ up to the highest investigated values, where we stopped at $\tan \beta=50$. $c_{\beta-\alpha}=0$, is allowed for all $\tan \beta$ values, with a roughly triangular shape, extending up to $c_{\beta-\alpha} \sim 0.2$.

The results for $\kappa_{\lambda}=\lambda_{h h h} / \lambda_{\mathrm{SM}}$ are presented in the lower plot of Fig. 8, with the total allowed area discussed above being now marked by the bounding black solid line. The red solid line indicates $\kappa_{\lambda} \equiv 1$. This is either the alignment limit for $c_{\beta-\alpha}=0$, or the "wrong sign limit" in the upper right corner. For the latter, see the discussion in Sect. 4.4. The color code shows the values reached by $\kappa_{\lambda}$. In the area allowed by all experimental and theoretical constraints, values of $\kappa_{\lambda} \lesssim 1$ are realized, going down to $\kappa_{\lambda} \sim-0.4$ in the "tip" to the right of the allowed area. The corresponding implications will be discussed in Sect. 5.4.

We now turn to the triple Higgs couplings involving at least one heavy Higgs boson. In Fig. 9 we show the results for $\lambda_{h h H}, \lambda_{h H H}, \lambda_{h A A}$ and $\lambda_{h H^{+} H^{-}}$in the upper left, upper right, lower left and lower right plot, respectively. As before, the area allowed by all experimental and theoretical constraints is indicated by a black solid line, and the color code shows the values reached by the triple Higgs couplings. In all four cases we find positive couplings with the minimum values reached for $c_{\beta-\alpha}=0$. The larger values are found in the right edge of the allowed area, with largest values (as in the case of $\lambda_{h h h}$ ) in the "tip" to the right of the allowed area. $\lambda_{h h H}$ is found to be larger around $\tan \beta \sim 8$ and $c_{\beta-\alpha} \sim 0.1$. The maximum values found for the rest of the triple Higgs couplings in this case are $\lambda_{h H H} \sim 12, \lambda_{h A A} \sim 12$ and $\lambda_{h H^{+} H^{-}} \sim 24$. It should be noted that here and in the following $\lambda_{h H^{+} H^{-}}$always reaches the maximum values of all the 
2HDM type I, scenario $\mathrm{C}, m_{12}^{2}=\left(m_{H}^{2} \cos ^{2} \alpha\right) /(\tan \beta)$
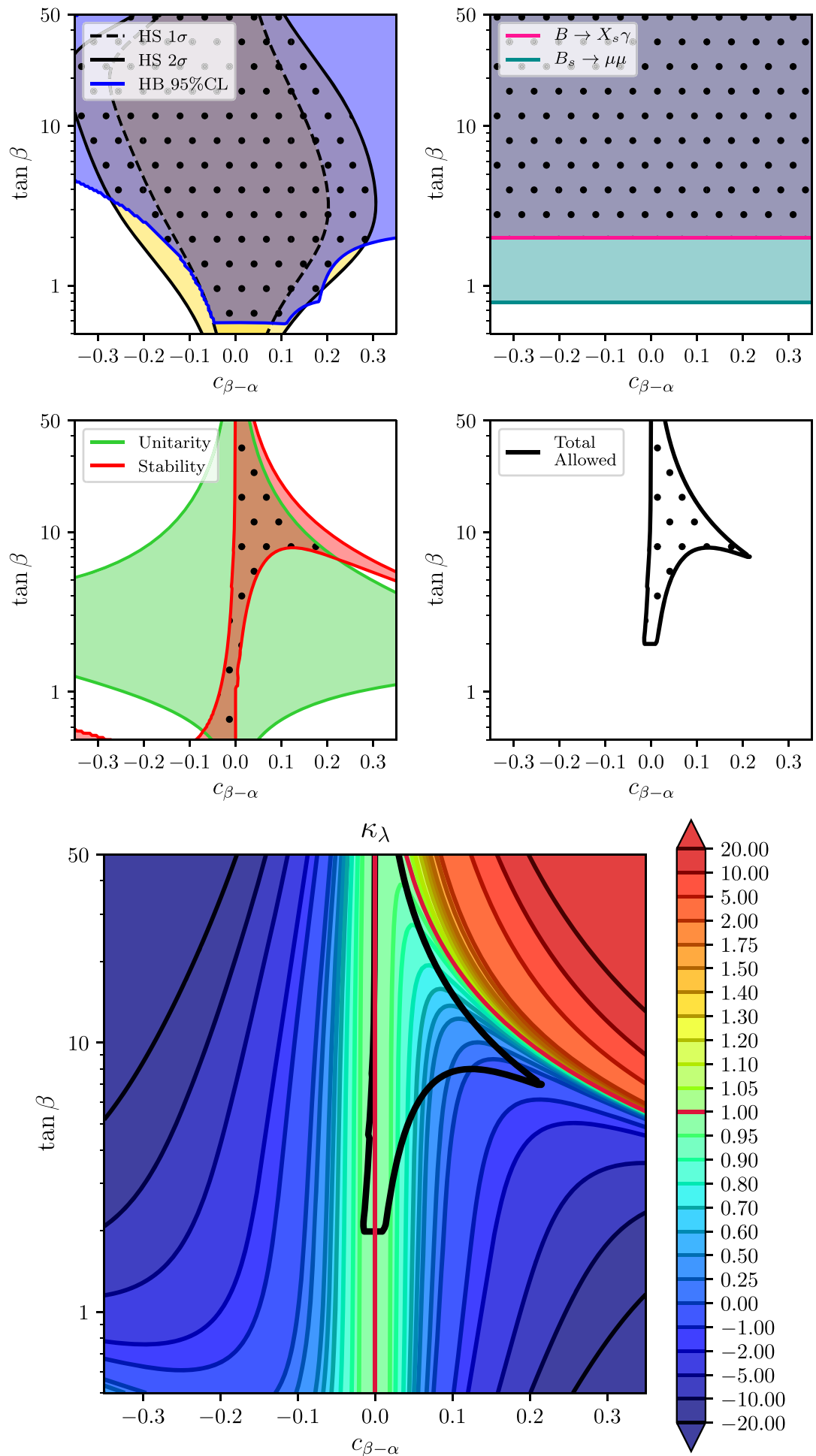

Fig. 8 Predictions for $\kappa_{\lambda}=\lambda_{h h h} / \lambda_{\text {SM }}$ in the 2HDM type I, scenario C, for $m_{H}=m_{A}=m_{H^{ \pm}}=1000 \mathrm{GeV}$ and $m_{12}^{2}=\left(m_{H}^{2} \cos ^{2} \alpha\right) /(\tan \beta)$ in the $\left(c_{\beta-\alpha}, \tan \beta\right)$ plane. Upper left plot: Allowed areas by direct searches at colliders (blue), constraints from the SM-like Higgs boson properties (yellow) and both (dotted). Upper right plot: Allowed areas by flavor physics from $B \rightarrow X_{s} \gamma$ (pink), $B_{s} \rightarrow \mu^{+} \mu-$ (teal) and both (dotted). Middle left plot: Allowed areas by the theoretical constraints from unitarity (green), stability (red) and both (dotted). Middle right plot: Total allowed area (dotted). Lower big plot: Contour lines of $\kappa_{\lambda}=\lambda_{h h h} / \lambda_{\mathrm{SM}}$. Red contours correspond to $\kappa_{\lambda}=1$. The thick solid contours is the boundary of the total allowed area 


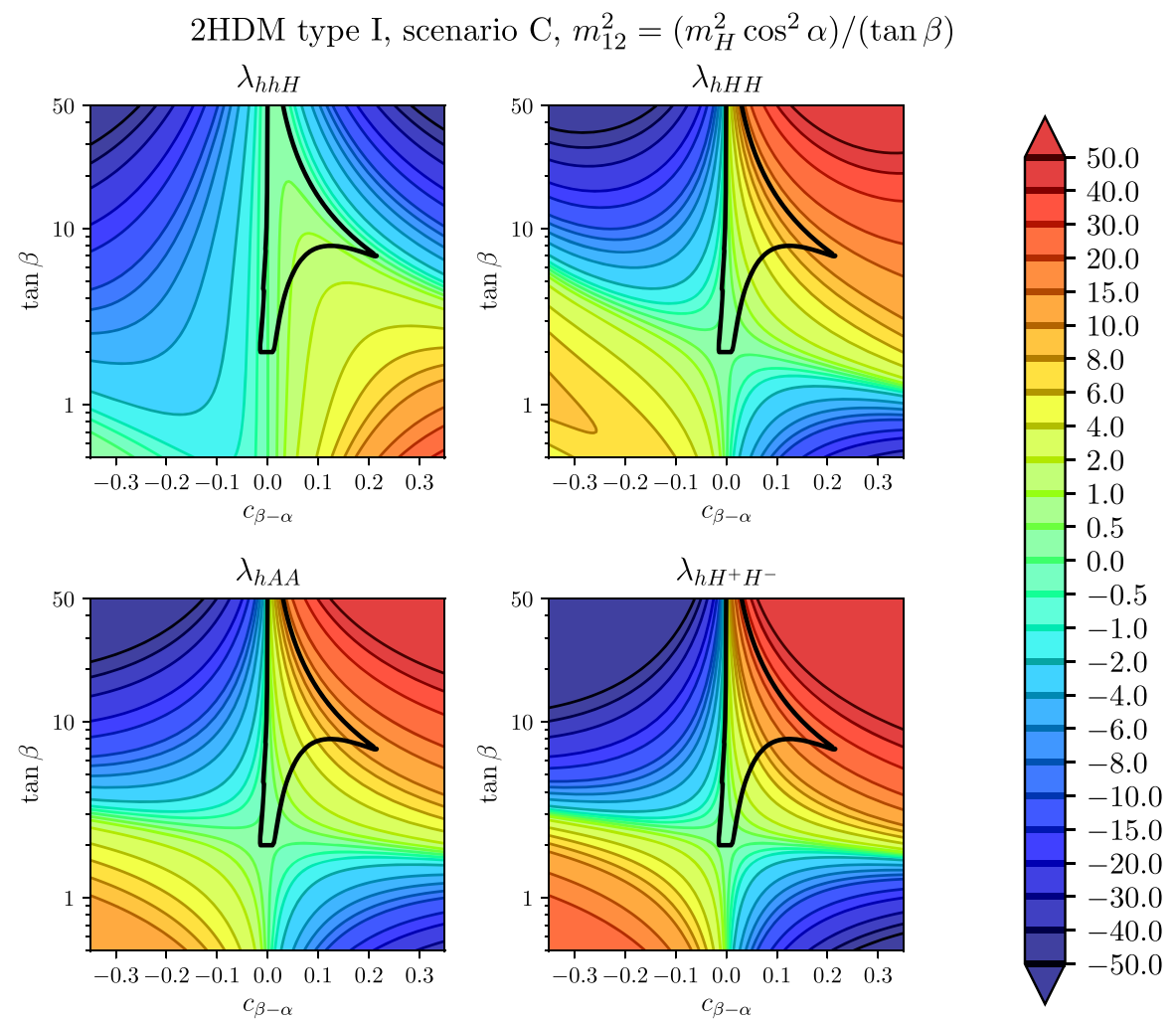

Fig. 9 Contour lines for triple Higgs couplings in the $2 \mathrm{HDM}$ type I, scenario C, for $m_{H}=m_{A}=m_{H^{ \pm}}=1000 \mathrm{GeV}$ and $m_{12}^{2}=$ $\left(m_{H}^{2} \cos ^{2} \alpha\right) /(\tan \beta)$ in the $\left(c_{\beta-\alpha}, \tan \beta\right)$ plane. Upper left: $\lambda_{h h H}$, upper right: $\lambda_{h H H}$, lower left: $\lambda_{h A A}$, lower right: $\lambda_{h H^{+} H^{-}}$. The thick solid contour is as in Fig. 8

considered triple Higgs boson couplings. The corresponding phenomenological implications will be discussed in Sect. 5.4.

We continue the exploration of scenario $\mathrm{C}$, type $\mathrm{I}$ in the $\left(c_{\beta-\alpha}, m_{12}^{2}\right)$ plane for $m_{H^{ \pm}}=m_{H}=m_{A}=650 \mathrm{GeV}$ and $\tan \beta=7.5$, as shown in Figs. 10 and 11. The sequence and the color coding of the plots is the same as in Figs. 8 and 9. The overall allowed area is restricted, particularly by the requirement of unitarity and stability, to be within a curved band around $m_{12}^{2}=55000 \mathrm{GeV}^{2}$, ranging from $c_{\beta-\alpha} \sim 0$ to $c_{\beta-\alpha} \sim 0.28$. Here the purple solid line in the middle left plot indicates that Eq. (29) is satisfied. The lower plot in Fig. 10 presents the results for $\kappa_{\lambda}$, which show a weak dependence on $m_{12}^{2}$. Values of $\kappa_{\lambda} \sim 1$ are found around $c_{\beta-\alpha}=0$ (as required by the alignment limit), but also around $c_{\beta-\alpha} \sim$ 0.26 . The lowest value of $\kappa_{\lambda} \sim 0.5$ is realized for $c_{\beta-\alpha}=$ 0.2 , whereas the highest value of $\kappa_{\lambda} \sim 1.2$ are found for $c_{\beta-\alpha} \sim 0.28$. Contrary to the $\left(c_{\beta-\alpha}, \tan \beta\right)$ plane shown in Fig. 8, we now also encounter values of $\kappa_{\lambda}$ larger than 1 . However, these are realized for the largest departure of the alignment limit, and thus will be under scrutiny by the next round of Higgs-boson rate measurements at the LHC.

The results for the triple Higgs couplings involving heavy Higgs bosons are shown in Fig. 11, analogous to Fig. 9. As for $\lambda_{h h h}$ the variation with $m_{12}^{2}$ (in the allowed inter- val) is relatively small. The intervals found in this case are $\lambda_{h h H} \sim[-1,0.3], \lambda_{h H H} \sim[-0.3,7], \lambda_{h A A} \sim[-0.3,7]$ and $\lambda_{h H^{+} H^{-}} \sim[-0.5,14]$. It should be noted that due to the contribution from $m_{12}^{2}$ here these couplings can also be slightly negative. As before, the maximum of $\lambda_{h h H}$ is found for $c_{\beta-\alpha} \sim 0.1$ whereas for the other couplings, which can be of $\mathcal{O}(10)$, the largest values are realized for the largest departure of the alignment limit, and thus will be under scrutiny by the next round of Higgs-boson rate measurements at the LHC.

We finish our analysis of the scenario C, type I in Figs. 12 and 13 , where we show the $\left(c_{\beta-\alpha}, m_{H}=m_{A}=m_{H^{ \pm}}\right)$ plane, and where $m_{12}^{2}$ is fixed by Eq. (29) to maximize the regions allowed by unitarity and stability of the potential, and with $\tan \beta=10$. The sequence and the color coding of the plots is the same as in Figs. 8 and 9. The upper left plot in Fig. 12 shows the areas allowed by HiggsBounds and HiggsSignals, as discussed in Sects. 4.3 and 4.4. The HiggsBounds allowed area exhibits several spikes around $m_{H}=m_{A}=m_{H^{ \pm}} \sim 400 \mathrm{GeV}$. Here the exclusion bounds are stemming from the channel $g g \rightarrow A \rightarrow$ $Z h \rightarrow l \bar{l} b \bar{b}$ [22], which exhibits several "spikes" which we identified as due to statistical fluctuations in the experimental limits. The overall allowed area now exhibits positive and 
2HDM type I, scenario C
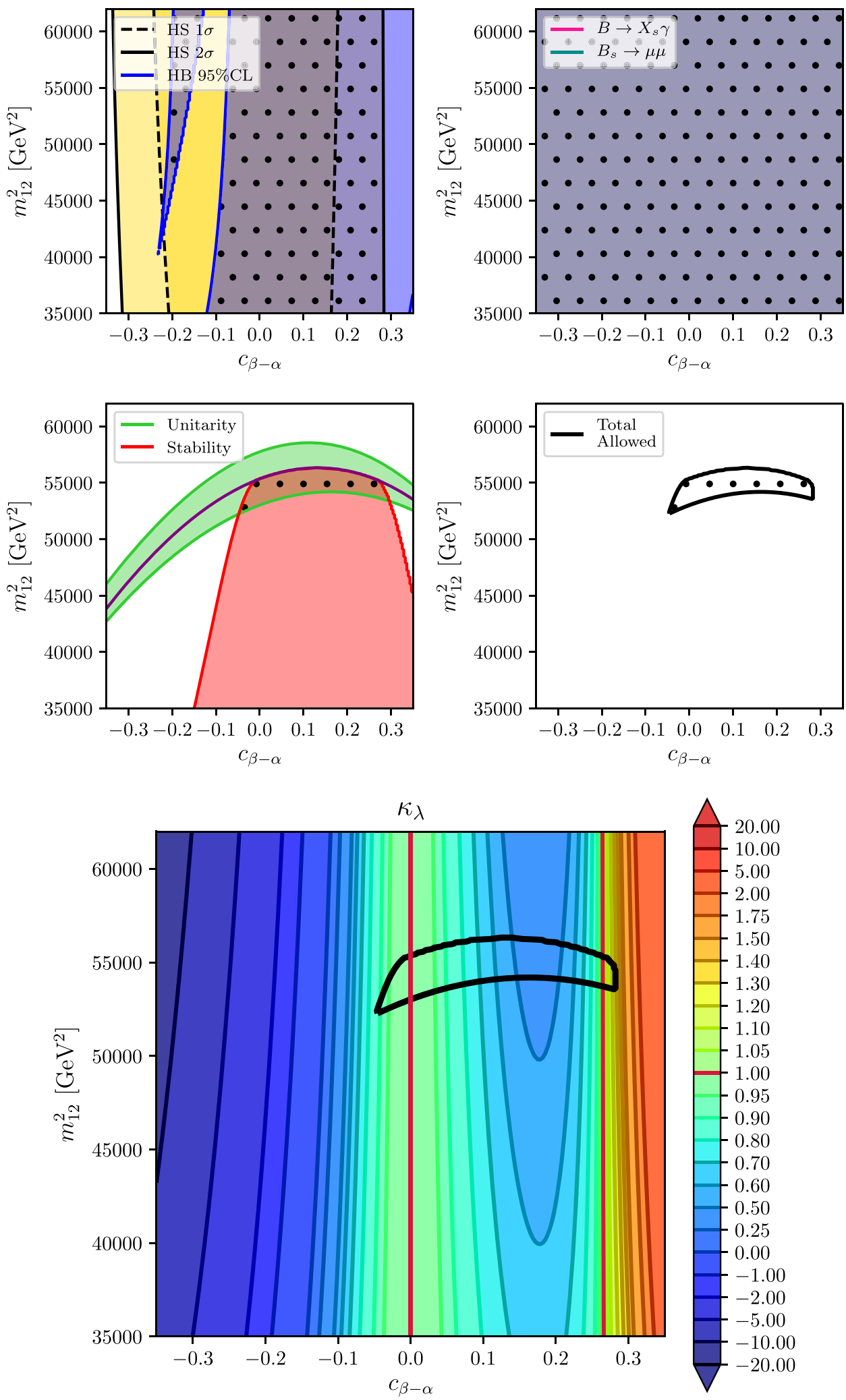

Fig. 10 Predictions for $\kappa_{\lambda}=\lambda_{h h h} / \lambda_{\mathrm{SM}}$ in the 2HDM type I, scenario C, in the $\left(c_{\beta-\alpha}, m_{12}^{2}\right)$ plane for $m_{H}=m_{A}=m_{H^{ \pm}}=650 \mathrm{GeV}$ and tan $\beta=$ 7.5. The description of the allowed regions is as in Fig. 8. Purple contour in the middle left plot satisfies the condition $m_{12}^{2}=\left(m_{H}^{2} \cos ^{2} \alpha\right) /(\tan \beta)$ 


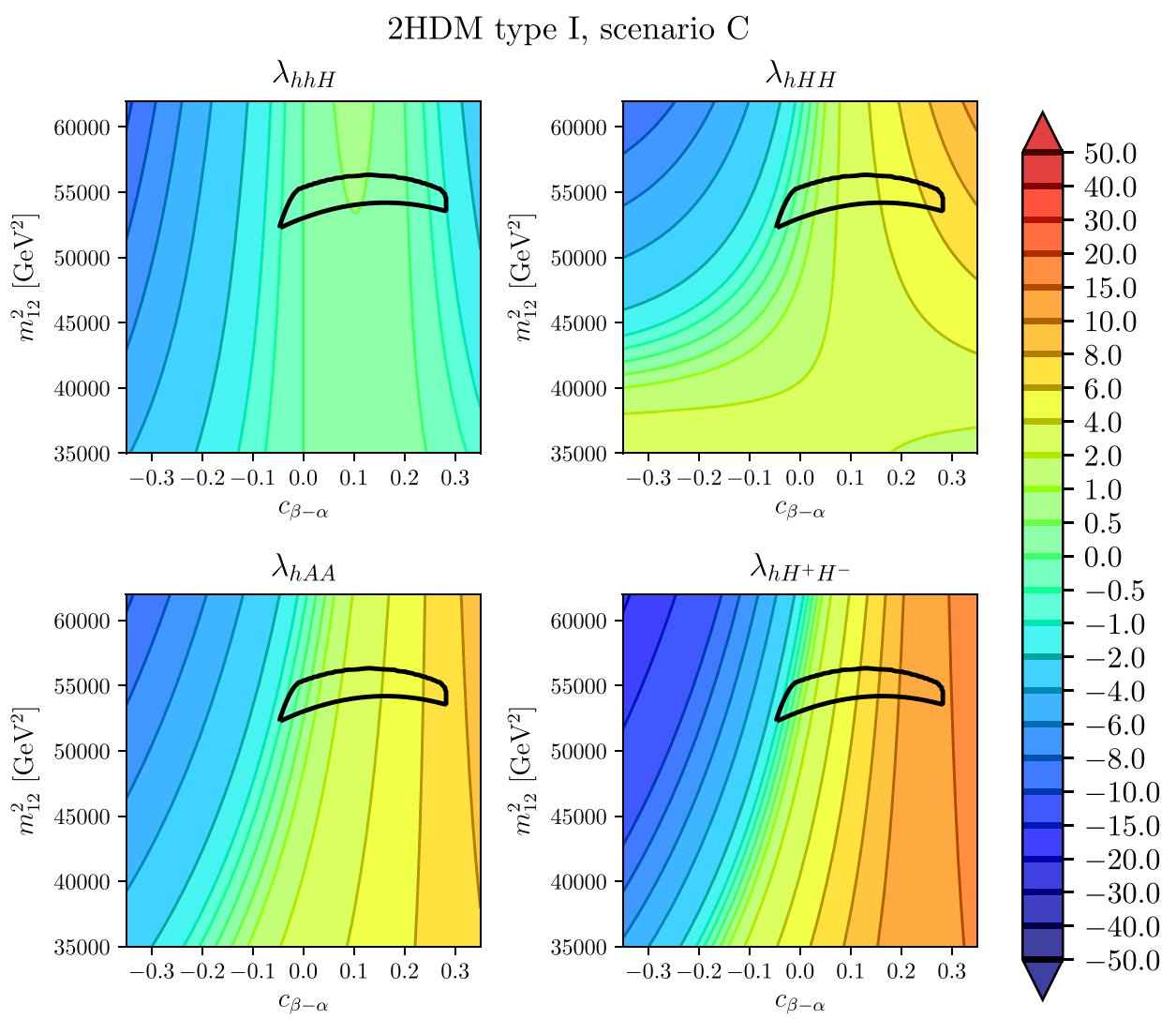

Fig. 11 Contour lines for triple Higgs couplings in the $2 \mathrm{HDM}$ type I, scenario C, in the $\left(c_{\beta-\alpha}, m_{12}^{2}\right)$ plane for $m_{H}=m_{A}=m_{H^{ \pm}}=650 \mathrm{GeV}$ and $\tan \beta=7.5$. Upper left: $\lambda_{h h H}$, upper right: $\lambda_{h H H}$, lower left: $\lambda_{h A A}$, lower right: $\lambda_{h H^{+} H^{-}}$. The thick solid contour is as in Fig. 10

negative values of $c_{\beta-\alpha}$ for low $m_{H^{ \pm}}<400 \mathrm{GeV}$. For larger masses only positive values are allowed, reaching slightly above $c_{\beta-\alpha} \sim 0.2$.

The values that can be reached by $\kappa_{\lambda}$, as shown in the lower plot of Fig. 12, range from $\kappa_{\lambda} \sim 0.07$ for $c_{\beta-\alpha} \sim 0.1$ and large $m_{H^{ \pm}}$close to $1200 \mathrm{GeV}$ to about $\kappa_{\lambda} \sim 1.2$ for the largest allowed $c_{\beta-\alpha}$ values and $m_{H^{ \pm}} \sim 300 \mathrm{GeV}$. The ranges reached by the triple Higgs couplings involving at least one heavy Higgs boson, as shown in Fig. 13, are found to be $\lambda_{h h H} \sim[-0.2,1.6], \lambda_{h H H} \sim[-0.2,12], \lambda_{h A A} \sim$ $[-0.2,12]$ and $\lambda_{h H^{+} H^{-}} \sim[-0.5,24]$. The largest values of $\lambda_{h h H}$ are found for $c_{\beta-\alpha} \sim 0.1$ and large $m_{H^{ \pm}}$and for the rest are found on the edge for larger $c_{\beta-\alpha}$ and $m_{H^{ \pm}} \gtrsim 800 \mathrm{GeV}$.

We finish our analysis of scenario $\mathrm{C}$ with the $\left(c_{\beta-\alpha}, m_{12}^{2}\right)$ plane in the 2HDM type II for $m_{H}=m_{A}=m_{H^{ \pm}}=$ $1000 \mathrm{GeV}$ and $\tan \beta=0.9$, as presented in Figs. 14 and 15. The sequence of the plots and the color coding are as in Figs. 10 and 11. The total allowed area is found, roughly between $c_{\beta-\alpha} \sim-0.05$ and $c_{\beta-\alpha} \lesssim 0.1$, as well as $m_{12}^{2} \gtrsim 2 \times 10^{5} \mathrm{GeV}^{2}$ and $m_{12}^{2} \lesssim 6 \times 10^{5} \mathrm{GeV}^{2}$.

The values that can be reached by $\kappa_{\lambda}$, as shown in the lower plot of Fig. 14, range from $\kappa_{\lambda} \sim 0.0$ for $c_{\beta-\alpha} \sim 0.13$ and low $m_{12}^{2}$ to $\kappa_{\lambda}=1$ for the alignment limit. The ranges reached by the triple Higgs couplings involving at least one heavy Higgs boson, as shown in Fig. 15, are found to be $\lambda_{h h H} \sim[-1,1.4], \lambda_{h H H} \sim[-0.2,12], \lambda_{h A A} \sim[-0.2,12]$ and $\lambda_{h H^{+} H^{-}} \sim[-0.4,24]$. Again negative values can be reached, due to the effects caused by $m_{12}^{2}$. The largest values for $\lambda_{h H H}, \lambda_{h H^{+} H^{-}}$and $\lambda_{h A A}$ are found for the lowest allowed $m_{12}^{2}$ values, and are nearly independent on $c_{\beta-\alpha}$. In contrast, $\lambda_{h h H}$ shows dependence on both variables where its maximum is found around $m_{12}^{2} \sim 400000 \mathrm{GeV}^{2}$ and $c_{\beta-\alpha} \sim 0.08$ and its minimum is found around $m_{12}^{2} \sim 550000 \mathrm{GeV}^{2}$ and $c_{\beta-\alpha} \sim-0.03$. As for the 2HDM type I, the phenomenological interpretation of these intervals will be given in Sect. 5.4.

\subsection{Scenario A}

We continue our numerical investigation by relaxing the conditions for the heavy Higgs-boson masses and evaluate the triple Higgs-boson couplings in scenario A, as defined in Sect. 4.1, $m_{A}=m_{H^{ \pm}} \neq m_{H},{ }^{4}$ and $m_{h}=125 \mathrm{GeV}$.

In Fig. 16 we present the $\left(m_{H^{ \pm}}=m_{A}, m_{H}\right)$ plane with $m_{12}^{2}=\left(m_{H}^{2} \cos ^{2} \alpha\right) /(\tan \beta)$, to maximize the parameter

\footnotetext{
${ }^{4}$ Here and in the following we will denote this common mass as $m_{H^{ \pm}}$.
} 
2HDM type I, scenario $\mathrm{C}, m_{12}^{2}=\left(m_{H}^{2} \cos ^{2} \alpha\right) /(\tan \beta)$
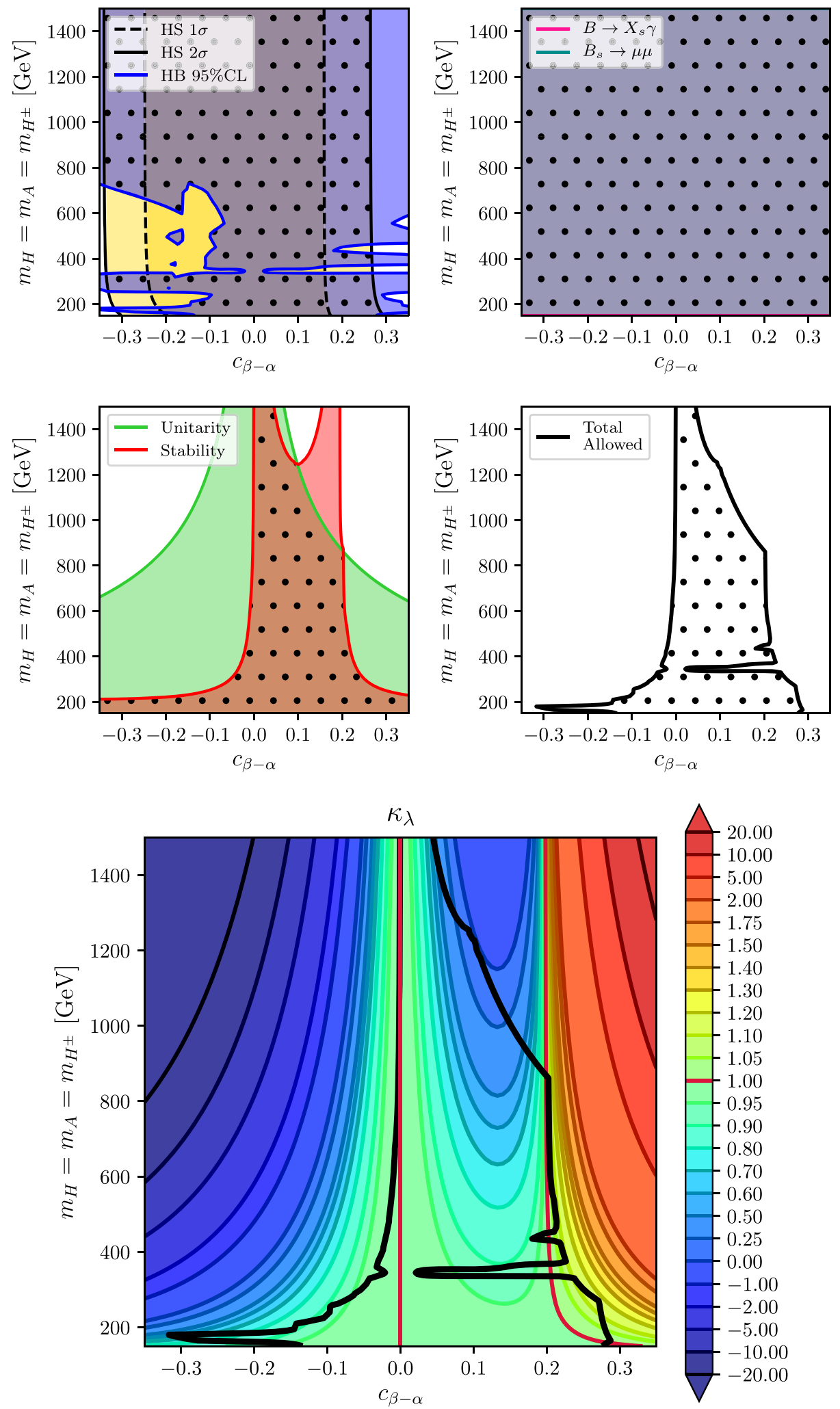

Fig. 12 Predictions for $\kappa_{\lambda}=\lambda_{h h h} / \lambda_{\mathrm{SM}}$ in the $2 \mathrm{HDM}$ type I, scenario C, in the $\left(c_{\beta-\alpha}, m\right)$ plane with $m_{=}=m_{H}=m_{A}=m_{H^{ \pm}}, m_{12}^{2}=$ $\left(m_{H}^{2} \cos ^{2} \alpha\right) /(\tan \beta)$ and $\tan \beta=10$. The description of the allowed regions is as in Fig. 8 


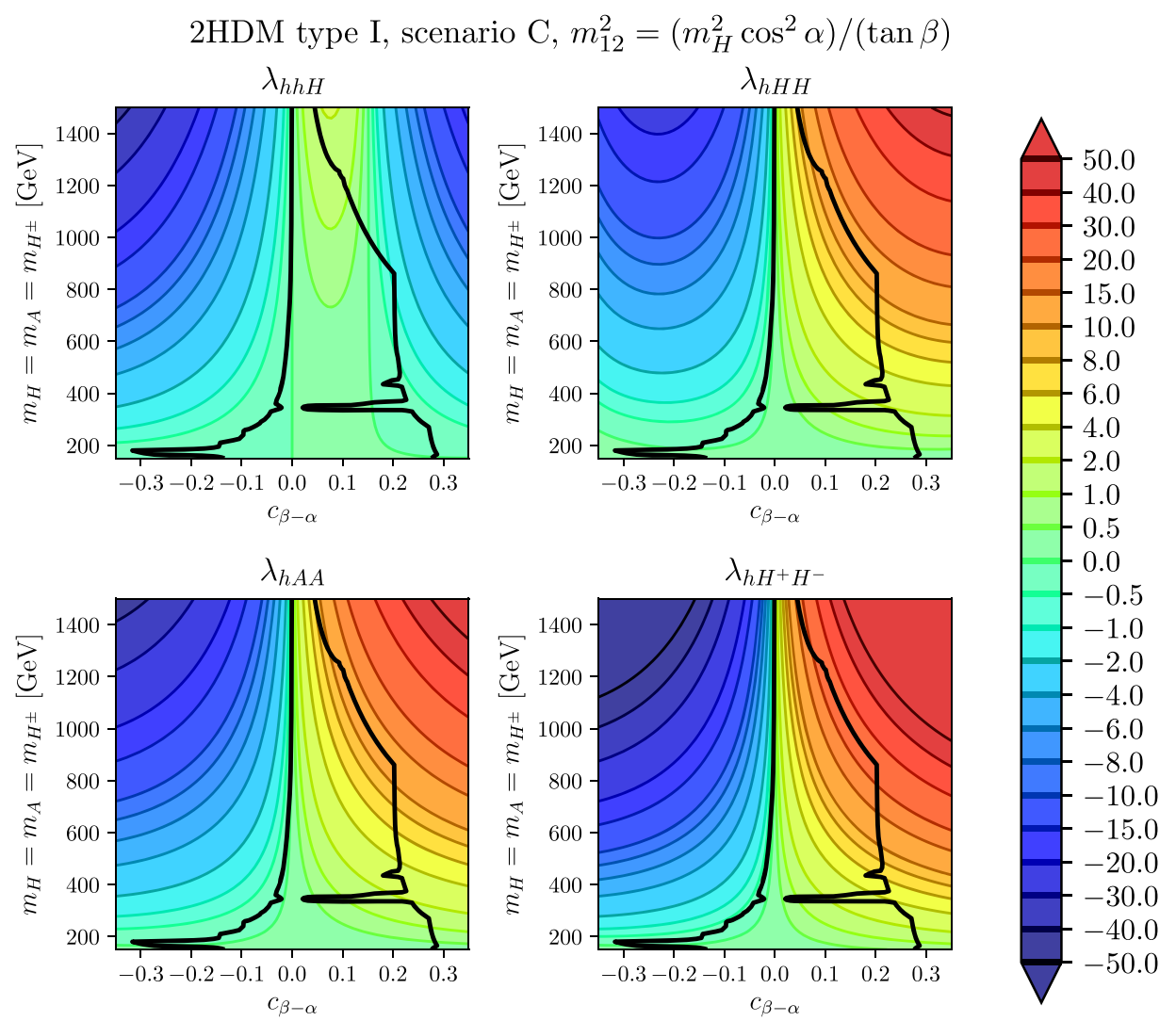

Fig. 13 Contour lines for triple Higgs couplings in the $2 \mathrm{HDM}$ type I, scenario C, in the $\left(c_{\beta-\alpha}, m\right)$ plane for $m=m_{H}=m_{A}=m_{H^{ \pm}}$, $m_{12}^{2}=\left(m_{H}^{2} \cos ^{2} \alpha\right) /(\tan \beta)$ and $\tan \beta=10$. Upper left: $\lambda_{h h H}$, upper right: $\lambda_{h H H}$, lower left: $\lambda_{h A A}$, lower right: $\lambda_{h H^{+} H^{-}}$. The thick solid contour is as in Fig. 12

space allowed by unitarity and stability of the Higgs potential, and $c_{\beta-\alpha}=0.2$ and $\tan \beta=10$. The upper two rows show the various constraints, with the same color coding as in Fig. 8. One can see that the LHC searches and measurements, as well as the flavor observables allow for the whole plane. Unitarity and stability roughly select a square bounded from above by $m_{H^{ \pm}} \sim m_{H} \sim 1000 \mathrm{GeV}$. The results for $\lambda_{h h h}$ are not explicitly shown, as they vary only very weakly in the chosen scenario. The values reached are in the interval $\kappa_{\lambda} \sim[0.98,1.02]$. The lower two rows in Fig. 16 show the results for the triple Higgs couplings involving at least one heavy Higgs boson. The upper left plot (of the two lower rows) shows $\lambda_{h h H}$, which is independent of $m_{H^{ \pm}}$. Lowest (highest) values are reached for high (low) values of $m_{H}$, following the analytic result in Eq. (33). They range from 0.02 to -1.5 .

The upper right plot depicts the results for $\lambda_{h H H}$, again independent of $m_{H^{ \pm}}$. Here lowest (highest) values are reached for low (high) values of $m_{H}$, following the analytic result in Eq. (35). For $\lambda_{h H H}$ they range from 0.2 to 16 . The lower row shows the results for $\lambda_{h A A}$ (left) and $\lambda_{h H^{+} H^{-}}$ (right), which exhibit a similar behavior, see Eq. (37) and Eq. (39). The values are nearly independent of $m_{H}$, where lowest (highest) values are found for low (high) $m_{A}=m_{H^{ \pm}}$. They range from 0 to 16 for $\lambda_{h A A}$, and from 0 to 32 for $\lambda_{h H^{+} H^{-}}$. As in Sect. 5.1 we leave the phenomenological discussion to Sect. 5.4.

Analogous results in the 2HDM type II are presented in Fig. 17, with the color codings as in Fig. 16. As before $m_{12}^{2}$ is fixed by $m_{12}^{2}=\left(m_{H}^{2} \cos ^{2} \alpha\right) /(\tan \beta)$. In order to maximize the results for the triple Higgs couplings we have chosen $c_{\beta-\alpha}=0.025$ and $\tan \beta=6.5$. The overall allowed region, as depicted in the upper two rows, can be found on the strip roughly around the diagonal $m_{H^{ \pm}}=m_{A} \sim m_{H}$. The results for $\lambda_{h h h}$ again vary only weakly in this region, and are found in the interval $\kappa_{\lambda} \sim[0.8,1]$. The third row shows the results for $\lambda_{h h H}$ (left) and $\lambda_{h H H}$ (right), which follow similar patterns and are independent of $m_{H^{ \pm}}$, see Eqs. (33) and (35). Lowest (highest) values are found at low (high) $m_{H}$, ranging from 0 to 1.25 for $\lambda_{h h H}$ and from 0.15 to 3 for $\lambda_{h H H}$. The fourth row presents the results for $\lambda_{h A A}$ (left) and $\lambda_{h H^{+} H^{-}}$ (right), which again follow similar patterns and are nearly independent of $m_{H}$, see Eqs. (37) and (39). The lowest values are found at the diagonal $m_{H^{ \pm}}=m_{A} \sim m_{H}$, whereas the highest values are found for the highest allowed $m_{H^{ \pm}}=$ $m_{A}>m_{H}$ with a mass splitting of about $250-300 \mathrm{GeV}$. 
2HDM type II, scenario C
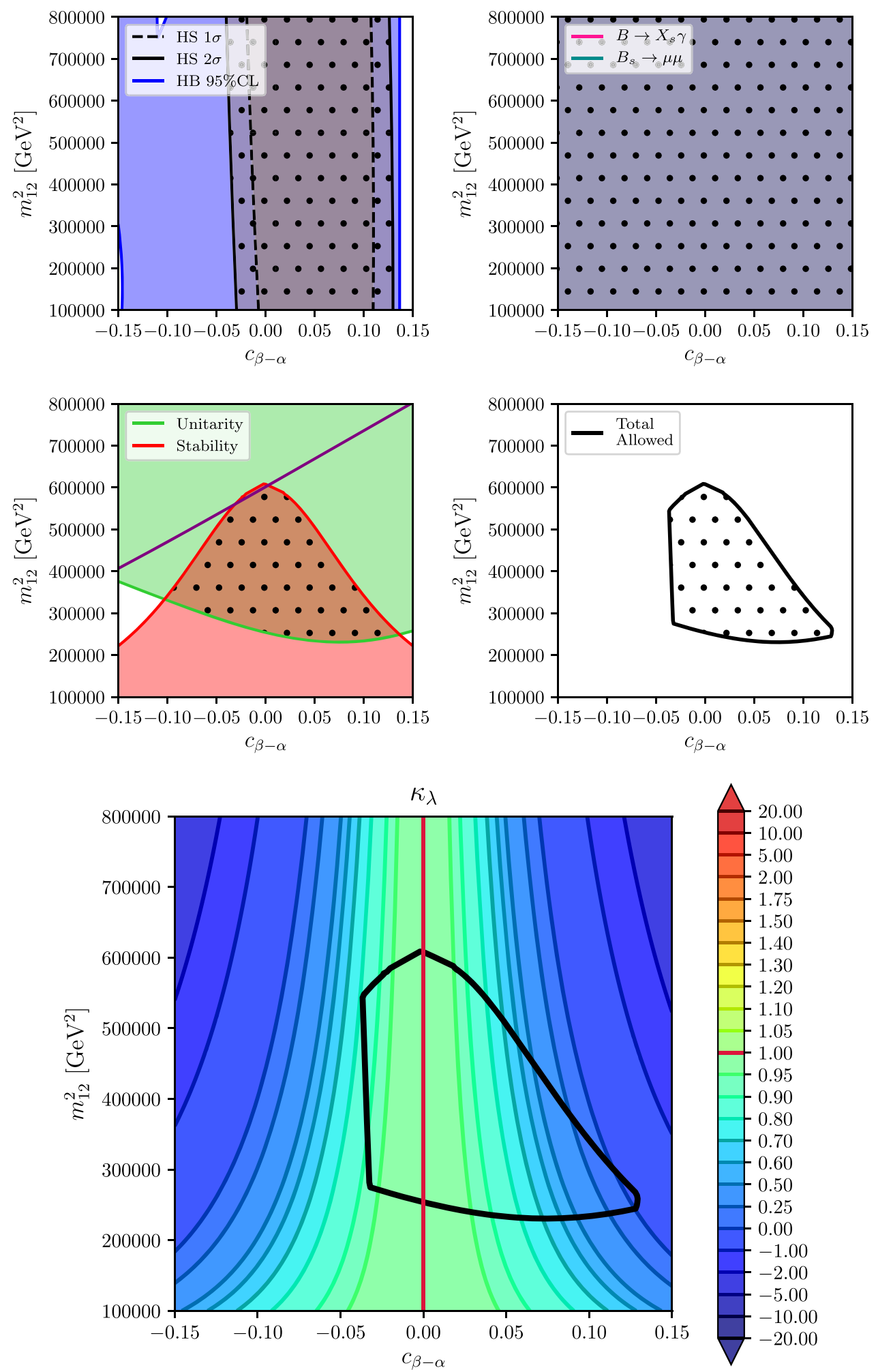

Fig. 14 Predictions for $\kappa_{\lambda}=\lambda_{h h h} / \lambda_{\mathrm{SM}}$ in the 2HDM type II, scenario $\mathrm{C}$, in the $\left(c_{\beta-\alpha}, m_{12}^{2}\right)$ plane with $m_{H}=m_{A}=$ $m_{H^{ \pm}}=1100 \mathrm{GeV}$ and $\tan \beta=0.9$. The description of the allowed regions is as in Fig. 8. Purple contour in the middle left plot satisfies the condition $m_{12}^{2}=\left(m_{H}^{2} \cos ^{2} \alpha\right) /(\tan \beta)$ 


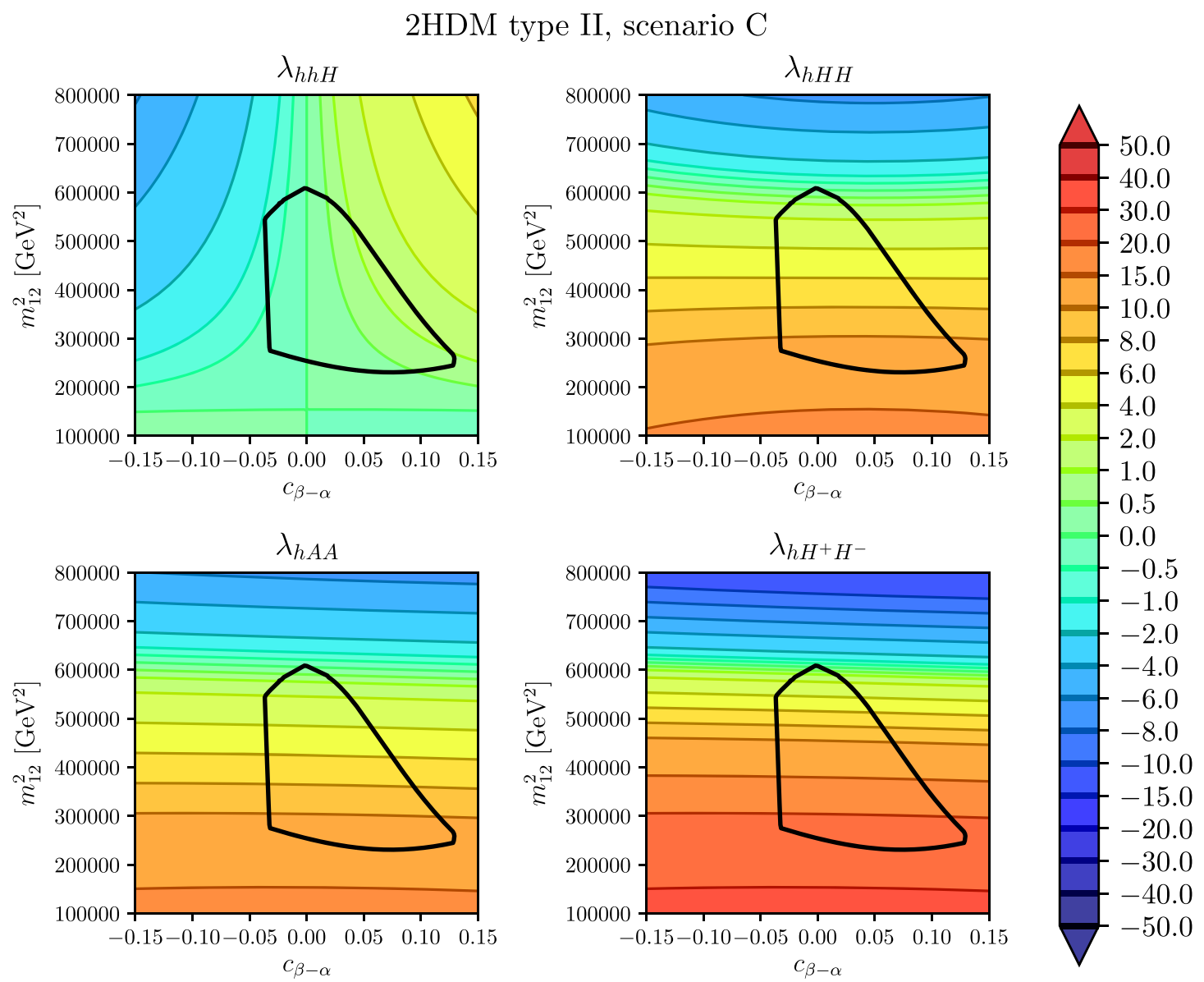

Fig. 15 Contour lines for triple Higgs couplings in the 2HDM type II, scenario C, in the $\left(c_{\beta-\alpha}, m_{12}^{2}\right)$ plane for $m_{H}=m_{A}=m_{H^{ \pm}}=1100 \mathrm{GeV}$, and $\tan \beta=0.9$. Upper left: $\lambda_{h h H}$, upper right: $\lambda_{h H H}$, lower left: $\lambda_{h A A}$, lower right: $\lambda_{h H^{+} H^{-}}$. The thick solid contour is as in Fig. 14

They range from 0.4 to 16 for $\lambda_{h A A}$ and from 0.8 to 32 for $\lambda_{h H^{+} H^{-}}$. The phenomenological implications are discussed in Sect. 5.4.

We finish our analysis in the scenario A with the 2HDM type II presented in Fig. 18, with the color codings as in Fig. 16. In comparison with the previous analysis we have chosen a relatively low value of $\tan \beta=0.9$, and fixed $m_{12}^{2}=100000 \mathrm{GeV}^{2}$, while for $c_{\beta-\alpha}$ a relatively large value (for the 2HDM type II) of $c_{\beta-\alpha}=0.05$ was chosen. The overall allowed region, as depicted in the upper two rows can be found roughly around $800 \mathrm{GeV}<m_{H^{ \pm}}=m_{A}<$ $1100 \mathrm{GeV}$ and $m_{H}$ between $500 \mathrm{GeV}$ and $1000 \mathrm{GeV}$. As before, the results for $\lambda_{h h h}$ vary only weakly in this region, and it takes values for $\kappa_{\lambda} \sim 0.9$ in the whole plane. The third row shows the results for $\lambda_{h h H}$ (left) and $\lambda_{h H H}$ (right), and as before both are independent of $m_{H^{ \pm}}$, see Eqs. (33) and (35). $\lambda_{h h H}$ exhibits a small variation between -0.14 to 0.23. $\lambda_{h H H}$, on the other hand, can reach very large values for large $m_{H}$, and it is found to be in the range of 0.3 and 15 . The fourth row presents the results for $\lambda_{h A A}$ (left) and $\lambda_{h H^{+} H^{-}}$ (right), which again follow similar patterns and are indepen- dent of $m_{H}$, see Eqs. (37) and (39). The lowest (highest) values are found at the lowest (highest) allowed values for $m_{H^{ \pm}}=m_{A} \sim 800$ (1100) GeV. They range from 8 to 16 for $\lambda_{h A A}$ and from 16 to 32 for $\lambda_{h H^{+} H^{-}}$. The phenomenological implications are discussed in Sect. 5.4.

\subsection{Scenario B}

We finish our numerical investigation with the third scenario suggested by the electroweak precision observables, scenario B, as defined in Sect. 4.1, $m_{A} \neq m_{H^{ \pm}}=m_{H}$, and $m_{h}=125 \mathrm{GeV}$.

In Fig. 19 we present the $\left(m_{H^{ \pm}}=m_{H}, m_{A}\right)$ plane with the other parameters chosen as in the corresponding scenario A, with $m_{12}^{2}=\left(m_{H}^{2} \cos ^{2} \alpha\right) /(\tan \beta)$ to maximize the parameter space allowed by unitarity and stability of the Higgs potential, and for $c_{\beta-\alpha}=0.2$ and $\tan \beta=10$. The upper two rows show the various constraints, with the same color coding as in Fig. 8. Besides, in the upper left plot,

\footnotetext{
${ }^{5}$ Here and in the following we will denote this common mass as $m_{H^{ \pm}}$.
} 
2HDM type I, scnario A, $m_{12}^{2}=\left(m_{H}^{2} \cos ^{2} \alpha\right) /(\tan \beta)$
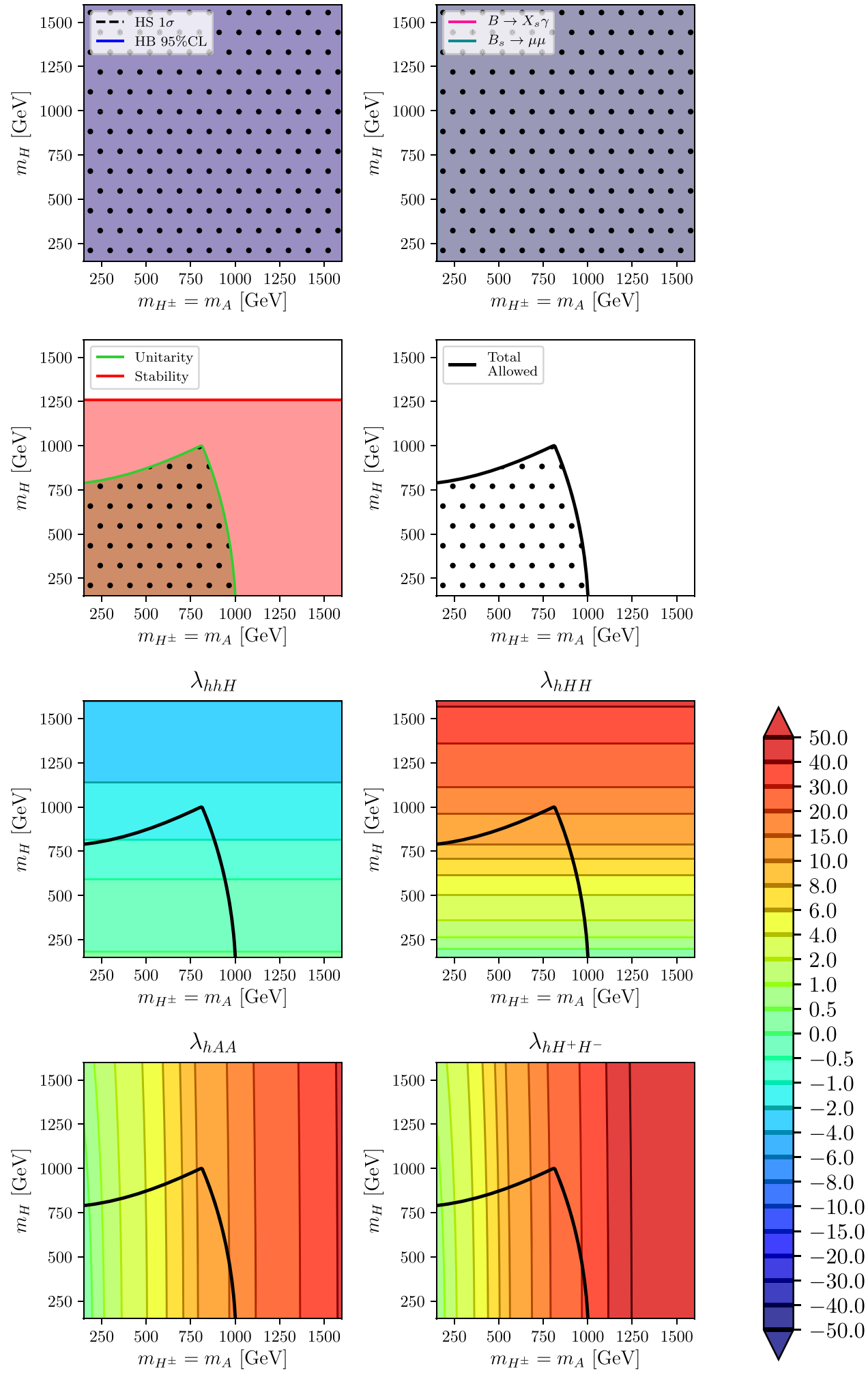

Fig. 16 Predictions for triple Higgs couplings in the 2HDM type I, scenario A, in the $\left(m_{H^{ \pm}}=m_{A}, m_{H}\right)$ plane with $c_{\beta-\alpha}=0.2, \tan \beta=10$ and $m_{12}^{2}=\left(m_{H}^{2} \cos ^{2} \alpha\right) /(\tan \beta)$. Upper four plots: allowed regions as in Fig. 8. Third line left: $\lambda_{h h H}$, third line right: $\lambda_{h H H}$, lower left: $\lambda_{h A A}$, lower right: $\lambda_{h H^{+} H^{-}}$ 
2HDM type II, scenario A, $m_{12}^{2}=\left(m_{H}^{2} \cos ^{2} \alpha\right) /(\tan \beta)$
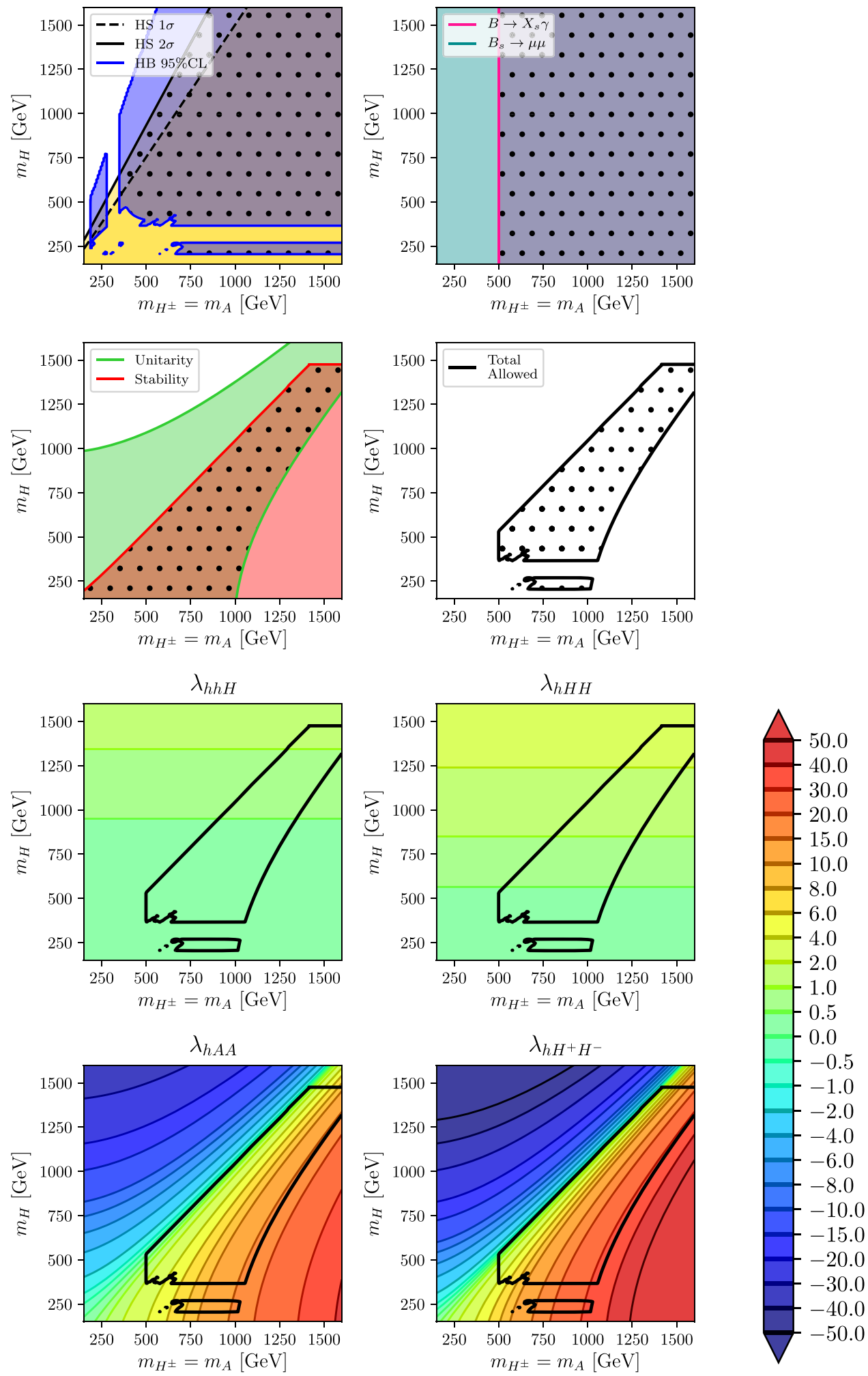

Fig. 17 Predictions for triple Higgs couplings in the 2HDM type II, scenario A, in the $\left(m_{H^{ \pm}}=m_{A}, m_{H}\right)$ plane with $c_{\beta-\alpha}=0.025, \tan \beta=$ 6.5 and $m_{12}^{2}=\left(m_{H}^{2} \cos ^{2} \alpha\right) /(\tan \beta)$. Upper four plots: allowed regions as in Fig. 8. Third line left: $\lambda_{h h H}$, third line right: $\lambda_{h H H}$, lower left: $\lambda_{h A A}$, lower right: $\lambda_{h H^{+} H^{-}}$ 
2HDM type II, scenario $\mathrm{A}, m_{12}^{2}=100000 \mathrm{GeV}^{2}$
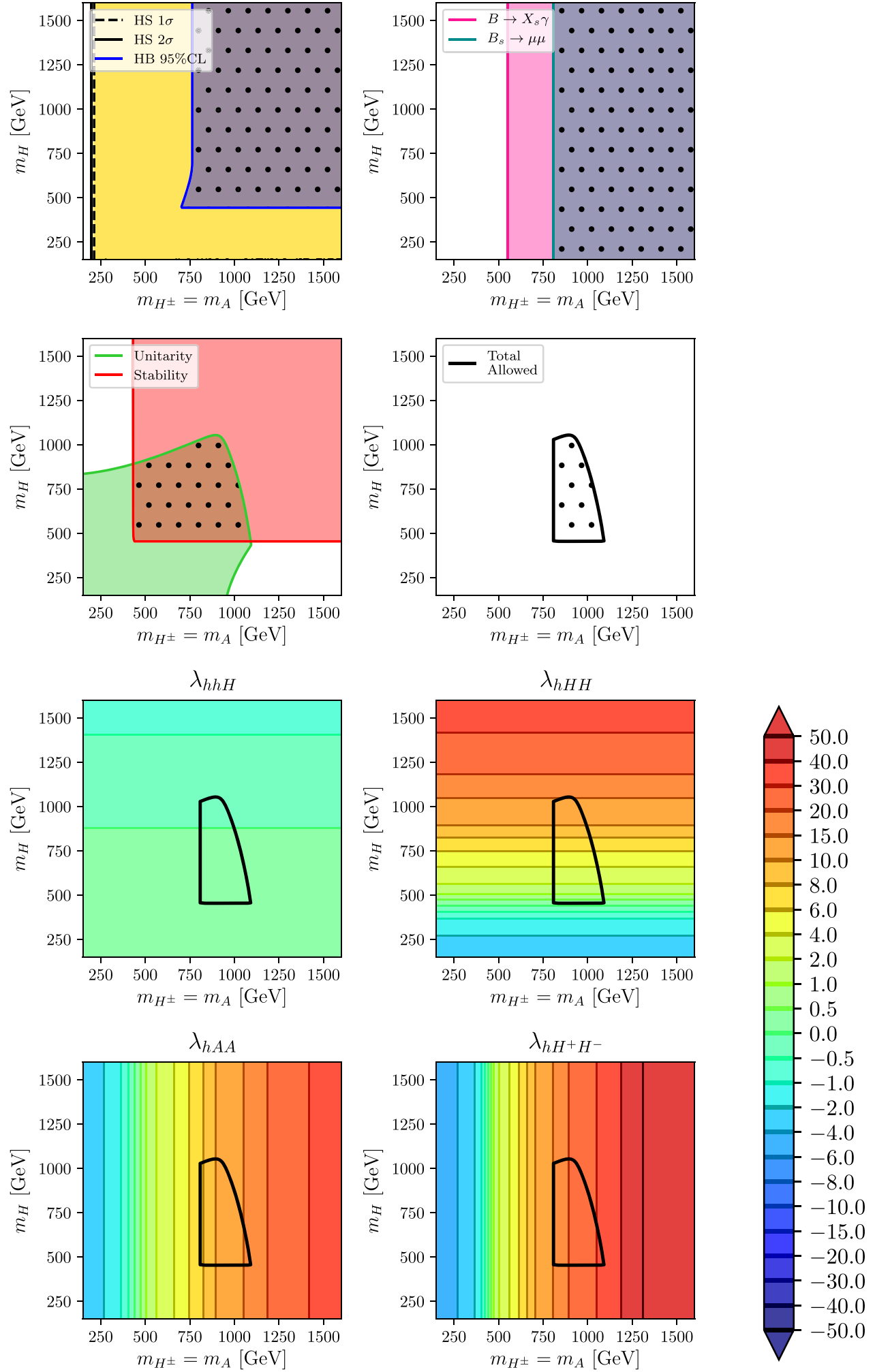

Fig. 18 Predictions for triple Higgs couplings in the 2HDM type II, scenario $\mathrm{A}$, in the $\left(m_{H^{ \pm}}=m_{A}, m_{H}\right)$ plane with $c_{\beta-\alpha}=0.05$, $\tan \beta=0.9$ and $m_{12}^{2}=100000 \mathrm{GeV}^{2}$. Upper four plots: allowed regions as in Fig. 8. Third line left: $\lambda_{h h H}$, third line right: $\lambda_{h H H}$, lower left: $\lambda_{h A A}$, lower right: $\lambda_{h H^{+} H^{-}}$ 
2HDM type I, scenario B, $m_{12}^{2}=\left(m_{H}^{2} \cos ^{2} \alpha\right) /(\tan \beta)$
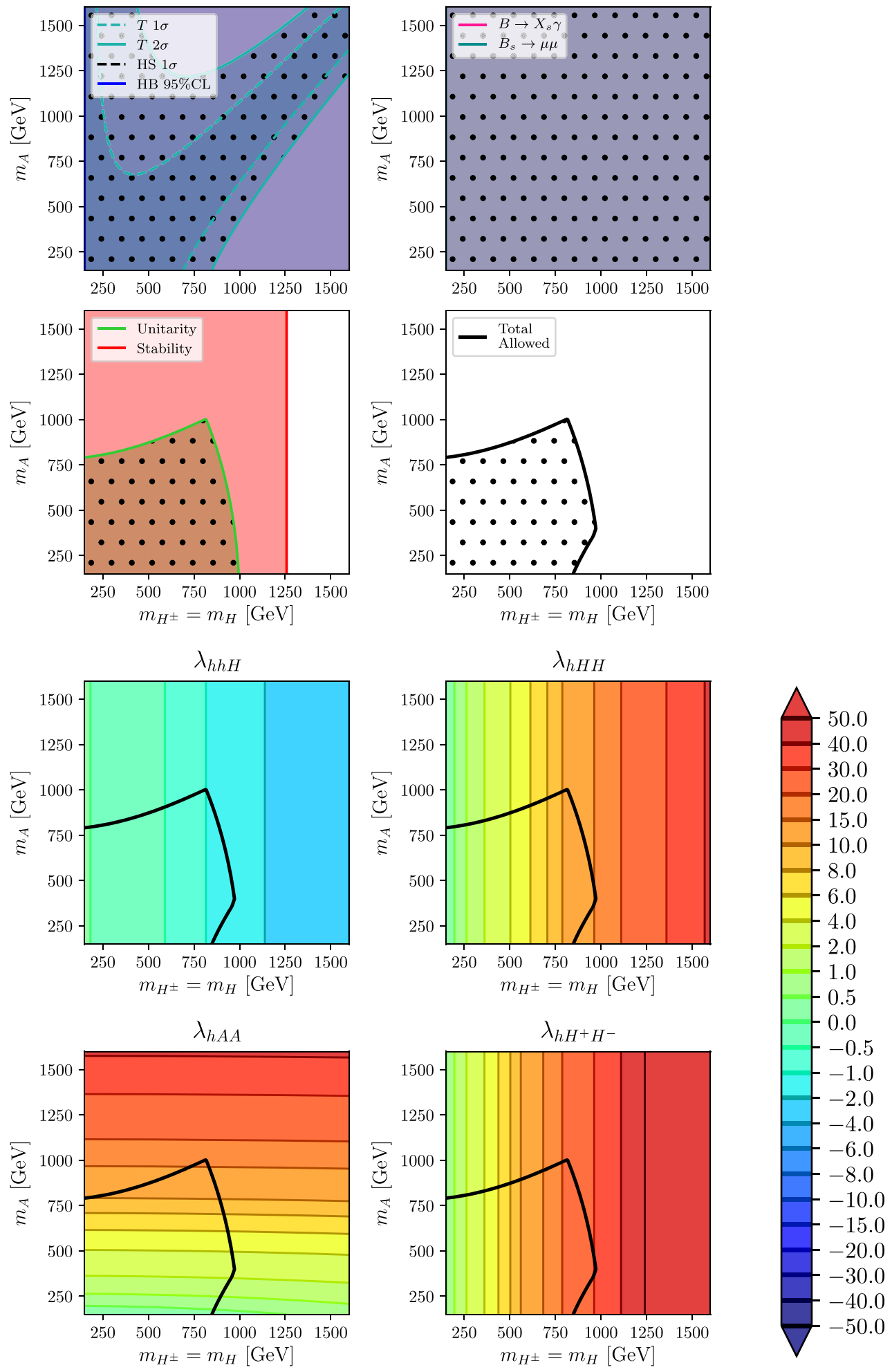

Fig. 19 Predictions for triple Higgs couplings in the 2HDM type I, scenario $\mathrm{B}$, in the $\left(m_{H^{ \pm}}=m_{H}, m_{A}\right)$ plane with $c_{\beta-\alpha}=0.2, \tan \beta=10$ and $m_{12}^{2}=\left(m_{H}^{2} \cos ^{2} \alpha\right) /(\tan \beta)$. Upper four plots: allowed regions as in Fig. 8. The light blue lines in the upper left plot correspond to the
$1 \sigma$ (dashed) and $2 \sigma$ (solid) allowed regions by the $T$ parameter. Third line left: $\lambda_{h h H}$, third line right: $\lambda_{h H H}$, lower left: $\lambda_{h A A}$, lower right: $\lambda_{h H^{+} H^{-}}$ 
2HDM type II, scenario $\mathrm{B}, m_{12}^{2}=100000 \mathrm{GeV}^{2}$
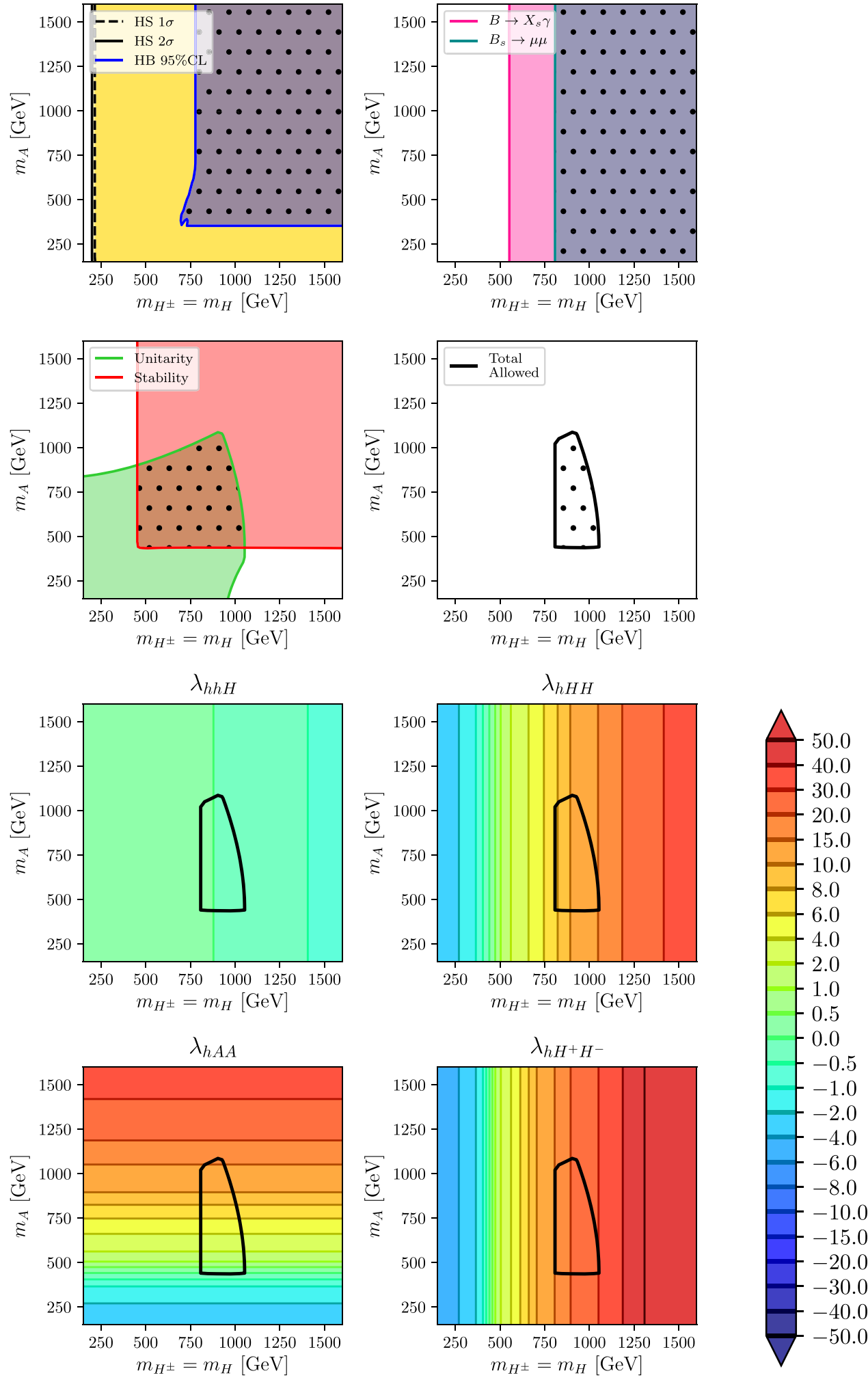

regions as in Fig. 8. Third line left: $\lambda_{h h H}$, third line right: $\lambda_{h H H}$, lower

left: $\lambda_{h A A}$, lower right: $\lambda_{h H^{+} H^{-}}$

Fig. 20 Predictions for triple Higgs couplings in the 2HDM type II, scenario $\mathrm{B}$, in the $\left(m_{H^{ \pm}}=m_{H}, m_{A}\right)$ plane with $c_{\beta-\alpha}=0.05$, $\tan \beta=0.9$ and $m_{12}^{2}=100000 \mathrm{GeV}^{2}$. Upper four plots: allowed 
where we indicate the regions allowed by the LHC measurements, we also indicate the bound arising from the EWPO, see Fig. 4. While the LHC measurements of the SM-like Higgs boson as well as the direct searches for BSM Higgs bosons do not yield restrictions in the parameter space, the EWPO favor a broad region roughly around the diagonal $m_{H^{ \pm}}=m_{H} \sim m_{A}$. As in the corresponding scenario A, unitarity and stability roughly select a square bounded from above by $m_{A} \sim m_{H} \sim 1000 \mathrm{GeV}$. The results for $\lambda_{h h h}$ are again not explicitly shown, as they vary only very weakly in the chosen scenario. The values reached are in the interval $\kappa_{\lambda} \sim[0.98,1.03]$. The lower two rows in Fig. 19 show the results for the triple Higgs couplings involving at least one heavy Higgs boson. The upper left plot (of the two lower rows) shows $\lambda_{h h H}$, which is independent of $m_{A}$. Lower (higher) values are reached for high (low) values of $m_{H^{ \pm}}$, following the analytic result in Eq. (33). They range from -1.5 to 1 . The upper right plot depicts the results for $\lambda_{h H H}$, again independent of $m_{A}$. Here, the lowest (highest) values are reached for the lowest (highest) allowed values of $m_{H^{ \pm}}$, following the analytic result in Eq. (35). For $\lambda_{h H H}$ they range from 0.2 to 15 . The lower row shows the results for $\lambda_{h A A}$ (left) and $\lambda_{h H^{+} H^{-}}$(right), where the latter exhibits a similar behavior as $\lambda_{h H H}$, see Eqs. (37) and (39). The values of $\lambda_{h A A}\left(\lambda_{h H^{+} H^{-}}\right)$are nearly independent of $m_{H^{ \pm}}\left(m_{A}\right)$, where the lowest (highest) values are found for the lowest (highest) allowed values of $m_{A}\left(m_{H^{ \pm}}=m_{H}\right)$. They range from 0.2 to 16 for $\lambda_{h A A}$, and from 0.5 to 30 for $\lambda_{h H^{+} H^{-}}$. As in Sect. 5.1, we leave the phenomenological discussion to Sect. 5.4.

The final scenario analyzed is scenario B analogous to the last example in scenario A as presented in Fig. 19, with the color codings as in Fig. 16. As in scenario A we have chosen a relatively low value of $\tan \beta=0.9$, and fixed $m_{12}^{2}=100000 \mathrm{GeV}^{2}$, while for $c_{\beta-\alpha}$ a relatively large value (for the 2HDM type II) of $c_{\beta-\alpha}=0.05$ was chosen. The overall allowed region, as depicted in the upper two rows can be found roughly around $m_{H^{ \pm}}=m_{H} \sim 900 \mathrm{GeV}$ and $m_{A}$ between $500 \mathrm{GeV}$ and $1000 \mathrm{GeV}$, analogous to the corresponding scenario A. As before, the results for $\lambda_{h h h}$ vary only weakly in this region, and it leads to values of $\kappa_{\lambda} \sim 0.9$ in the whole plane. The lower two rows in Fig. 20 show the results for the triple Higgs couplings involving at least one heavy Higgs boson. The upper left plot (of the two lower rows) shows $\lambda_{h h H}$, which is independent of $m_{A}$ and varies only weakly in the parameter plane. Lower (higher) values are reached for high (low) values of $m_{H^{ \pm}}$, following the analytic result in Eq. (33). They range from -0.14 to 0 . The upper right plot depicts the results for $\lambda_{h H H}$, again independent of $m_{A}$. Here, the lowest (highest) values are reached for the lowest (highest) allowed values of $m_{H^{ \pm}}$, following the analytic result in Eq. (35). For $\lambda_{h H H}$ they range from 8 to 15 . The lower row shows the results for $\lambda_{h A A}$ (left) and $\lambda_{h H^{+} H^{-}}$(right), where the latter exhibits a similar behav- ior as $\lambda_{h H H}$, see Eqs. (37) and (39). The values of $\lambda_{h A A}$ $\left(\lambda_{h H^{+} H^{-}}\right)$are independent of $m_{H^{ \pm}}\left(m_{A}\right)$, where the lowest (highest) values are found for the lowest (highest) allowed values of $m_{A}\left(m_{H^{ \pm}}=m_{H}\right)$. They range from 0.14 to 16 for $\lambda_{h A A}$, and from 15 to 30 for $\lambda_{h H^{+} H^{-}}$.

Finally, to close the numerical results section, we present in Table 3 some examples of interesting configurations that maximize the size of the triple Higgs couplings. In the Yukawa type I, all examples have $\tan \beta>1$ while for type II all the points are around $\tan \beta \sim 1$. This is mainly due to the constraints from the LHC data, because it is easier to accommodate a SM-like Higgs in those regions (see Sect. 4.3). In addition, particularly in type I, flavor observables disallow low values of $\tan \beta$. In type I we recover a larger allowed parameter region by choosing $m_{12}^{2}$ according to Eq. (29), especially for the larger values of $\tan \beta$, which are easier in conflict with the theoretical constraints, as we discussed in Sect. 4.2. Furthermore, in type II the tight constraint from $B \rightarrow X_{s} \gamma$ that sets $m_{H^{ \pm}} \gtrsim 500 \mathrm{GeV}$ should be also satisfied. However, as we have discussed in the previous sections, the main constraint that prevent from obtaining large triple Higgs couplings are the theoretical constraints.

For both types, I and II, points with larger triple Higgs couplings are also the ones with the heavier Higgs masses around $1 \mathrm{TeV}$ (where we have not explored values above $\sim 1.6 \mathrm{TeV}$ ). The only exception to this are $\lambda_{h h h}$ and $\lambda_{h h H}$. In the case of $\lambda_{h h h}$ in the alignment limit the SM value is reproduced. On the other hand, $\lambda_{h h H}$ is proportional to $c_{\beta-\alpha}$, see "Appendix A". Consequently, their extrema are both found outside the alignment limit. As a consequence those points are stronger tested and possibly "easier" excluded in the future by more precise measurements. Overall, and particularly for type II, $\kappa_{\lambda}$ is close to unity. While this does not correspond to large enhancements of di-Higgs production, the deviations are still large enough to be tested at future colliders, see Sect. 3. On the other hand, it is possible to find large allowed values of couplings involving more than one heavy Higgs boson near the alignment limit. Those triple Higgs couplings always have positive and large values for all scenarios A, B and C. In fact, they can be larger in scenarios $\mathrm{A}$ and $\mathrm{B}$ due to the allowed splitting of two of the masses, as we have seen in Sects. 5.2 and 5.3. In those cases, the larger mass is the one of the Higgs boson that appears in the vertex. Overall, we find values of $\mathcal{O}(10)$, with the maximum value corresponding to $\lambda_{\mathrm{hH}^{+} \mathrm{H}^{-}} \sim 30$ in both type I and II.

In Table 3 we also include points with smaller Higgs masses that also yield interesting sizes of the triple Higgs couplings. Due to the relatively smaller masses, these points are better kinematically accessible. These last kind of points are presumably the easiest to probe at future colliders. For these more moderate, but potentially more accessible masses, we find triple Higgs couplings with half the size w.r.t. the maximum values. 
Table 3 Examples in the 2HDM for parameter inputs that present large size of some triple Higgs couplings allowed by current constraints in the Yukawa type I (top table) and Yukawa type II (bottom table). Values in bold are the ones that are close to the maximum deviation from the SM for $\kappa_{\lambda}$ and the absolute extremum for the other couplings found in our study. In each table, horizontal lines distinguish between scenario C (top), scenario A (center) and scenario B (bottom) defined in Sect. 4.1. The masses $m_{H}, m_{A}$ and $m_{H^{ \pm}}$are expressed in $\mathrm{GeV}$ and $m_{12}^{2}$ is expressed in $\mathrm{GeV}^{2}$. Points marked with an asterisk (*) are also allowed in type II

\begin{tabular}{|c|c|c|c|c|c|c|c|c|c|c|}
\hline$m_{H}$ & $m_{A}$ & $m_{H^{ \pm}}$ & $\tan \beta$ & $c_{\beta-\alpha}$ & $m_{12}^{2}$ & $\kappa_{\lambda}$ & $\lambda_{h h H}$ & $\lambda_{h H H}$ & $\lambda_{h A A}$ & $\lambda_{h H^{+} H^{-}}$ \\
\hline \multicolumn{11}{|c|}{ Yukawa type I } \\
\hline 750 & 750 & 750 & 5.5 & 0.25 & Eq. (29) & -0.4 & 0.4 & 7 & 6 & 12 \\
\hline 1000 & 1000 & 1000 & 7.5 & 0.2 & Eq. (29) & -0.3 & 0.1 & 13 & 12 & 24 \\
\hline 650 & 650 & 650 & 6.0 & 0.2 & Eq. (29) & 0.1 & 0.5 & 4 & 4 & 8 \\
\hline 300 & 300 & 300 & 15.0 & 0.25 & Eq. (29) & 1.5 & -0.6 & 2 & 2 & 5 \\
\hline 400 & 400 & 400 & 12.5 & 0.2 & 12500 & 1.2 & -0.4 & 3 & 3 & 6 \\
\hline 600 & 600 & 600 & 10.0 & 0.2 & Eq. (29) & 1.0 & -0.5 & 6 & 6 & 12 \\
\hline$* 1500$ & 1500 & 1500 & 2.0 & -0.025 & 820000 & 0.8 & -1.2 & 3 & 3 & 6 \\
\hline 650 & 400 & 400 & 12.0 & 0.15 & Eq. (29) & 0.9 & -0.3 & 6 & 2 & 4 \\
\hline 300 & 600 & 600 & 2.5 & 0.1 & 5000 & 1.0 & 0.0 & 1 & 6 & 12 \\
\hline 300 & 600 & 600 & 12.5 & 0.2 & Eq. (29) & 1.1 & -0.2 & 2 & 6 & 12 \\
\hline$* 700$ & 1200 & 1200 & 2.0 & 0.0 & Eq. (29) & 1.0 & 0.0 & 0.0 & 16 & 32 \\
\hline 700 & 1000 & 700 & 7.0 & 0.2 & Eq. (29) & 0.3 & 0.2 & 6 & 14 & 11 \\
\hline 350 & 600 & 350 & 10.0 & 0.2 & Eq. (29) & 1.0 & -0.1 & 2 & 6 & 4 \\
\hline 600 & 350 & 600 & 10.0 & 0.2 & Eq. (29) & 1.0 & -0.5 & 6 & 2 & 11 \\
\hline \multicolumn{11}{|c|}{ Yukawa type II } \\
\hline 1100 & 1100 & 1100 & 0.9 & 0.13 & 260000 & -0.1 & 0.9 & 11 & 11 & 23 \\
\hline 1500 & 1500 & 1500 & 0.8 & 0.05 & 775000 & 0.5 & 1.7 & 11 & 11 & 21 \\
\hline 600 & 600 & 600 & 1.5 & 0.02 & 25000 & 1.0 & 0.0 & 5 & 5 & 10 \\
\hline 1150 & 1000 & 1000 & 0.95 & 0.025 & 210000 & 1.0 & 0.1 & 15 & 10 & 19 \\
\hline 400 & 600 & 600 & 1.5 & 0.04 & 10000 & 1.0 & 0.0 & 2 & 6 & 11 \\
\hline 1350 & 1000 & 1350 & 0.9 & 0.05 & 460000 & 0.7 & 0.8 & 15 & 1 & 30 \\
\hline 600 & 400 & 600 & 1.5 & 0.05 & 8000 & 1.0 & -0.1 & 6 & 2 & 12 \\
\hline
\end{tabular}

Finally, before ending this section we would like to make some comments about the viability of our 2HDM scenarios to produce a strong first order EW phase transition (FOPT), which is needed for EW Baryogenesis. In [71] it has been pointed out that in the 2HDM a FOPT is correlated with large values of the Higgs couplings $\lambda_{4}$ and/or $\lambda_{5}$. This issue of the preferred 2HDM Higgs self couplings favoring a FOPT and the correlated heavy Higgs mass region for $m_{A}$ and $m_{H}$ together with the preferences in the other relevant $2 \mathrm{HDM}$ parameters like $c_{\beta-\alpha}$, $\tan \beta$ and $m_{12}^{2}$ have also been explored in $[72,73]$. In order to study the strength of the EW phase transition for our 2HDM scenarios, which exhibit large Higgs self-couplings, we follow [73]. Instead of the more standard method using the thermal 1-loop effective potential (as done, for instance, in [72]) the method of [73] correlates this strength with the zero temperature vacuum energy difference of the $2 \mathrm{HDM}$ with respect to the SM. Finding this difference, $\Delta \mathcal{F}_{0}=\mathcal{F}_{0}-\mathcal{F}_{0}^{\mathrm{SM}}$, usually normalised to the SM value $\Delta \mathcal{F}_{0} / \mathcal{F}_{0}^{\mathrm{SM}}$, to be in the range $\Delta \mathcal{F}_{0} / \mathcal{F}_{0}^{\mathrm{SM}} \leq-0.34$ provides, accordingly to [73], a good indicator of a FOPT. Since this topic is clearly beyond the scope of this work, we have just evaluated here this estimate of $\Delta \mathcal{F}_{0} / \mathcal{F}_{0}^{\mathrm{SM}}$ for the points listed in Table $3 .^{6}$ We find that all the points in this table give large negative values (except the ones in the third and seventh rows leading to differences of around -0.23) fulfilling $\Delta \mathcal{F}_{0} / \mathcal{F}_{0}^{\mathrm{SM}} \leq-0.34$. Therefore, we conclude that our 2HDM scenarios leading to large Higgs self-couplings appear to favor a FOPT.

\subsection{Possible implications for future collider measurements}

We now turn to the phenomenological implications of the allowed ranges found for the various triple Higgs couplings, as discussed in the previous sections. As an overall result we

\footnotetext{
${ }^{6}$ We warmly thank Jose Miguel No for his invaluable help in our investigation of the FOPT, and for providing us with his private code for the estimate of $\Delta \mathcal{F}_{0} / \mathcal{F}_{0}^{\text {SM }}$.
} 
find that the allowed intervals for the various triple Higgs couplings depend only weakly on the chosen EWPO scenario A, $\mathrm{B}$ or C. However, the 2HDM type I exhibits a substantially stronger variation in $\lambda_{h h h}$ than type II. This is mostly owed to the larger allowed deviation from the alignment limit, see Sects. 4.3, 4.4. In this section we will concentrate on the anticipated impact of the triple Higgs couplings on the various di-Higgs production cross sections, where we leave a full phenomenological analysis for future work [74].

For $\lambda_{h h h}$ we roughly find allowed intervals of $[-0.5,1.5]$ in the 2HDM type I and $[0,1]$ in type II. While the production of two SM-like Higgs bosons, both at $p p$ and at $e^{+} e^{-}$colliders depends already at the tree-level on $\lambda_{h h h}$ and $\lambda_{h h H}$, the dependence on $\lambda_{h h h}$ is expected to be substantially stronger due to the propagator suppression with the inverse of $m_{H}^{2}$ of $\lambda_{h h H}$. Consequently, over the possible parameter range of $\lambda_{h h h}$ the HL-LHC is not expected to yield a precision on $\kappa_{\lambda}$ better than $35 \%$, and a deviation from $\lambda_{h h h}=0$ can not be established better than $\sim 2 \sigma$. Comparing the HL-LHC to the ILC500, the HL-LHC performs better (worse) than the ILC500 for $\kappa_{\lambda} \lesssim(\gtrsim) 0.5$, where both intervals are still allowed in both types of the 2HDM. In other words, the HLLHC results in comparison with the ILC500 may look a bit better than anticipated for $\kappa_{\lambda}=1$. However, in this comparison it must be kept in mind that the HL-LHC analysis is based on the variation of the Higgs triple coupling only, whereas for the ILC500 (at $\kappa_{\lambda}=1$ ) it has been shown that the analysis holds also for a variation of all Higgs-boson couplings within their anticipated experimental accuracies. Furthermore, deviations below $\kappa_{\lambda} \sim 0.5$ are realized for larger deviations from the alignment limit and may thus be tested in the next round of Higgs rate measurements at the LHC. Combining the ILC500 measurements with the final stage of the ILC1000, the Linear Collider shows a substantially better result than the HL-LHC for all the allowed $\lambda_{h h}$ parameter space. Only around a vanishing trilinear Higgs coupling similar precisions are anticipated (but the above mentioned caveat of the differences in the HL-LHC and ILC analyses still holds).

The phenomenological implications of the allowed ranges for $\lambda_{h h H}$ are twofold. This coupling can enhance or suppress the contribution of the off-shell heavy Higgs in the $h h$ production, which, however, are generally suppressed as mentioned above. On the other hand, a very large enhancement of this coupling would yield a relatively large cross section for $h H$ production. However, we find that large values of $\lambda_{h h H}$ are not allowed taking all existing experimental and theoretical constraints into account.

The triple Higgs couplings involving two heavy Higgs bosons, $\lambda_{h H H}, \lambda_{h A A}$ and $\lambda_{h H^{+} H^{-}}$can have a very strong impact on the heavy di-Higgs production and possibly facilitate the discovery of such heavier Higgs bosons (see, e.g., $[54,70])$. Roughly independent of the EWPO scenario and the 2HDM type, we find values of up to 15, 16 and 32, respectively. Here it must be kept in mind that the larger values of a triple Higgs coupling of $h$ to two heavy Higgs bosons are realized for larger values of the respective heavy Higgs-boson mass. Consequently, the effects of the large coupling and the heavy mass always go in opposite directions. A detailed study will be left to future work [74].

Finally, we would like to comment briefly about the projections after HL-LHC. As we have discussed above, the requirement of having the properties of the light $\mathcal{C P}$-even Higgs-boson, $h$, in agreement with the LHC rate measurements, see Sect. 4.4, restricts in particular the possible deviation of the Higgs-boson sector from the alignment limit. The Higgs-boson rate measurements will improve significantly at the HL-LHC [65]. If the HL-LHC does not find any significant deviation from the SM predictions, this would restrict further the possible deviations from the alignment limit and thus in particular the deviations of $\kappa_{\lambda}$ from unity (such an analysis, however, goes beyond the scope of our paper). If, on the other hand, the HL-LHC would observe a deviation from the SM predictions, a new fit around the then preferred values would have to be performed. The then possible sizes of the triple Higgs couplings will strongly depend on the hypothetical future preferred (non-SM) Higgs-boson sector parameters (again, such an analysis goes far beyond the scope of our paper).

\section{Conclusions}

An important task at future colliders is the measurement of the triple Higgs coupling $\lambda_{h h h}$. Depending on its size relative to the SM value, certain collider options result in a higher experimental accuracy. Similarly, large values of triple Higgs couplings involving heavy Higgs bosons can lead to enhanced production cross sections of BSM Higgs bosons.

Within the framework of Two Higgs Doublet Models (2HDM) type I and II we investigate the allowed ranges for all triple Higgs couplings involving at least one light, SMlike Higgs boson. We take into account all relevant theoretical and experimental constraints. From the theory side these comprise unitarity, and stability conditions. From the experimental side we require agreement with the direct BSM Higgsboson searches, as well as with measurements of the SM-like Higgs-boson rate as measured at the LHC. We furthermore require agreement with flavor observables and electroweak precision data (where the $T$ parameter plays the most important role). In this context we investigate more extensively the dependence of several of these constraints on the soft $Z_{2}$-breaking parameter, $m_{12}^{2}$. Here we find that large values of this parameter can affect notably the allowed parameter space, especially in the region of large $\tan \beta$. 
For theoretical constraints $m_{12}^{2}$ plays a key role: lower (higher) values are favored by the tree-level stability (unitarity) constraint, and the size of the intersection region is thus controlled by $m_{12}^{2}$. Thus, to enlarge the allowed region by both unitarity and stability we have used Eq. (29) on several occasions.

Regarding the experimental constraints, BSM Higgs boson searches and measurements of the $125 \mathrm{GeV}$ Higgs boson at the LHC can also be sensitive to the effects of $m_{12}^{2}$ in the scalar sector such like the $h \rightarrow \gamma \gamma$ decay (via the $h H^{+} H^{-}$vertex) or the production of a heavy BSM boson that decays to two $125 \mathrm{GeV}$ Higgs bosons, specially in the range of low masses. On the other hand, the triple Higgs couplings $\lambda_{h H^{+} H^{-}}$and $\lambda_{H H^{+} H^{-}}$also enter in the 2HDM prediction for $B_{S} \rightarrow \mu^{+} \mu^{-}$via the $h$ and $H$ Higgs penguins contributions with charged Higgs bosons in the loops, and they can be relevant (see also [33]). The largest effect from $m_{12}^{2}$ in $B_{S} \rightarrow \mu^{+} \mu^{-}$is due to $\lambda_{H H^{+} H^{-}}$in the region of large $\tan \beta$ and low $m_{H^{ \pm}}$and, therefore, this region is correspondingly constrained by the $B_{s} \rightarrow \mu^{+} \mu^{-}$data.

Based on a parameter scan we investigated several mass and parameter planes. We demanded agreement with the above given constraints and evaluated the maximum and minimum values of the various triple Higgs couplings. For the SM-type triple Higgs coupling w.r.t. its SM value, $\kappa_{\lambda}=$ $\lambda_{h h h} / \lambda_{\mathrm{SM}}$, we roughly find allowed intervals of $[-0.5,1.5]$ in the 2HDM type I and $[0,1]$ in type II. The production of two SM-like Higgs bosons, both at $p p$ and at $e^{+} e^{-}$colliders depends already at the tree-level strongly on $\lambda_{h h h}$. Consequently, over the possible parameter range of $\lambda_{h h h}$ the HLLHC is not expected to yield a precision on $\kappa_{\lambda}$ better than $35 \%$, and a deviation from $\lambda_{h h h}=0$ can not be established better than $\sim 2 \sigma$. Comparing the HL-LHC to the ILC500, the HL-LHC performs better (worse) than the ILC500 for $\kappa_{\lambda} \lesssim(\gtrsim)$ 0.5. Combining the ILC500 measurements with the final stage of the ILC1000, the Linear Collider shows a substantially better result than the HL-LHC for all the values of $\lambda_{h h h}$ in the allowed intervals that we have found.

The production of two light Higgs bosons can also depend on $\lambda_{h h H}$ in the 2HDM. In this case, the prediction in the alignment limit is $\lambda_{h h H}=0$, but here we reach the maximum (minimum) value around $c_{\beta-\alpha}= \pm 0.05$. We find that the total allowed interval of this coupling is $[-1.4,1.5]$ for type I and $[-1.6,1.8]$ for type II.

Concerning the triple Higgs couplings involving two heavy 2HDM Higgs bosons, we find large allowed values for both 2HDM type I and II. For $\lambda_{h H H}, \lambda_{h A A}$ and $\lambda_{h H^{+} H^{-}}$ we find maximum values of up to 15,16 and 32 , respectively. These triple Higgs couplings can have a very strong impact on the heavy di-Higgs production at $p p$ and $e^{+} e^{-}$colliders. Large coupling values can possibly facilitate the discovery of such heavier Higgs bosons. However, it must be kept in mind that the larger values of triple Higgs couplings of $h$ with two heavy Higgs bosons are realized for larger values of the respective heavy Higgs-boson mass. Consequently, the effects of the large coupling and the heavy mass always go in opposite directions. A detailed analysis of the various production cross sections will be analyzed elsewhere [74].

Acknowledgements We thank T. Stefaniak for assistance with HiggsBounds and HiggsSignals. We also thank A. Arnan and A. Pich for helpful discussions regarding the correct implementation of the Higgs penguin contributions in $B_{s} \rightarrow \mu^{+} \mu^{-}$. The present work has received financial support from the 'Spanish Agencia Estatal de Investigación" (AEI) and the EU "Fondo Europeo de Desarrollo Regional" (FEDER) through the project FPA2016-78022-P and from the grant IFT Centro de Excelencia Severo Ochoa SEV-2016-0597. The work of S.H. was also supported in part by the MEINCOP Spain under contract FPA2016-78022-P and in part by the "Spanish Red Consolider MultiDark" FPA2017-90566-REDC. This work has also received partial funding/support from the European Union's Horizon 2020 research and innovation programme under the Marie Skłodowska-Curie grant agreement No 674896. The work of F.A. was also supported by the Spanish Ministry of Science and Innovation via a FPU grant with code FPU18/06634.

Data Availability Statement This manuscript has no associated data or the data will not be deposited. [Authors' comment: All data is contained in the article, no additional data is available.]

Open Access This article is licensed under a Creative Commons Attribution 4.0 International License, which permits use, sharing, adaptation, distribution and reproduction in any medium or format, as long as you give appropriate credit to the original author(s) and the source, provide a link to the Creative Commons licence, and indicate if changes were made. The images or other third party material in this article are included in the article's Creative Commons licence, unless indicated otherwise in a credit line to the material. If material is not included in the article's Creative Commons licence and your intended use is not permitted by statutory regulation or exceeds the permitted use, you will need to obtain permission directly from the copyright holder. To view a copy of this licence, visit http://creativecomm ons.org/licenses/by/4.0/. Funded by SCOAP ${ }^{3}$.

\section{A Feynman rules}

In this appendix we present the Feynman rules, obtained with the Mathematica package [75], of the considered triple Higgs couplings in the $\lambda_{i}$ basis, defined in Eq. (4), and in the physical basis, defined in Eq. (13). The relation of these Feynman rules with the dimensionless couplings $\lambda_{h h_{i}} h_{j}$ that have been studied in this work is given in Eq. (16).

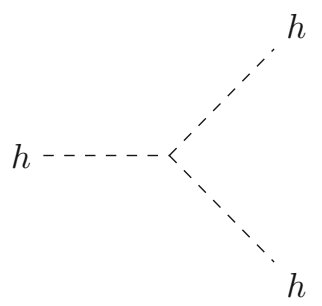


$\lambda_{i}$ basis:

$$
=3 i v\left\{\lambda_{1} c_{\beta} s_{\alpha}^{3}-\lambda_{2} c_{\alpha}^{3} s_{\beta}+\left(\lambda_{3}+\lambda_{4}+\lambda_{5}\right)\left(c_{\alpha}^{2} c_{\beta} s_{\alpha}-c_{\alpha} s_{\alpha}^{2} s_{\beta}\right)\right\} .
$$

Physical basis:

$$
\begin{aligned}
= & -\frac{3 i}{v}\left\{m_{h}^{2} s_{\beta-\alpha}^{3}+\left(3 m_{h}^{2}-2 \bar{m}^{2}\right) c_{\beta-\alpha}^{2} s_{\beta-\alpha}\right. \\
& \left.+2 \cot 2 \beta\left(m_{h}^{2}-\bar{m}^{2}\right) c_{\beta-\alpha}^{3}\right\} .
\end{aligned}
$$

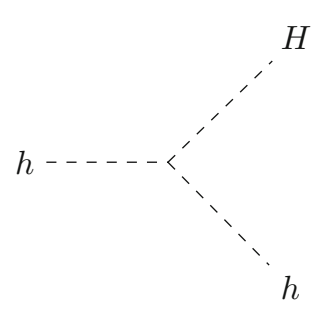

$\lambda_{i}$ basis:

$$
\begin{aligned}
= & -i v\left\{3 \lambda_{1} c_{\alpha} c_{\beta} s_{\alpha}^{2}+3 \lambda_{2} c_{\alpha}^{2} s_{\alpha} s_{\beta}\right. \\
& \left.+\left(\lambda_{3}+\lambda_{4}+\lambda_{5}\right)\left(c_{\alpha}^{3} c_{\beta}-2 c_{\alpha}^{2} s_{\alpha} s_{\beta}-2 c_{\alpha} c_{\beta} s_{\alpha}^{2}+s_{\alpha}^{3} s_{\beta}\right)\right\} .
\end{aligned}
$$

Physical basis:

$$
\begin{aligned}
= & \frac{i c_{\beta-\alpha}}{v}\left\{\left(2 m_{h}^{2}+m_{H}^{2}-4 \bar{m}^{2}\right) s_{\beta-\alpha}^{2}\right. \\
& +2 \cot 2 \beta\left(2 m_{h}^{2}+m_{H}^{2}-3 \bar{m}^{2}\right) s_{\beta-\alpha} c_{\beta-\alpha} \\
& \left.-\left(2 m_{h}^{2}+m_{H}^{2}-2 \bar{m}^{2}\right) c_{\beta-\alpha}^{2}\right\} .
\end{aligned}
$$

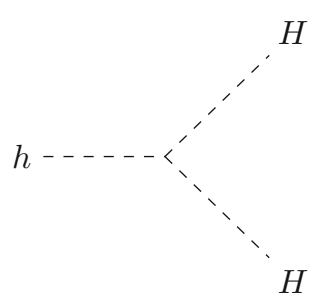

$\lambda_{i}$ basis:

$$
\begin{aligned}
= & i v\left\{3 \lambda_{1} c_{\alpha}^{2} c_{\beta} s_{\alpha}-3 \lambda_{2} c_{\alpha} s_{\alpha}^{2} s_{\beta}\right. \\
& \left.+\left(\lambda_{3}+\lambda_{4}+\lambda_{5}\right)\left(-c_{\alpha}^{3} s_{\beta}-2 c_{\alpha}^{2} c_{\beta} s_{\alpha}+2 c_{\alpha} s_{\alpha}^{2} s_{\beta}+c_{\beta} s_{\alpha}^{3}\right)\right\} .
\end{aligned}
$$

Physical basis:

$$
=-\frac{i s_{\beta-\alpha}}{v}\left\{\left(m_{h}^{2}+2 m_{H}^{2}-2 \bar{m}^{2}\right) s_{\beta-\alpha}^{2}\right.
$$

$$
\begin{aligned}
& +2 \cot 2 \beta\left(m_{h}^{2}+2 m_{H}^{2}-3 \bar{m}^{2}\right) c_{\beta-\alpha} s_{\beta-\alpha} \\
& \left.-\left(m_{h}^{2}+2 m_{H}^{2}-4 \bar{m}^{2}\right) c_{\beta-\alpha}^{2}\right\} .
\end{aligned}
$$

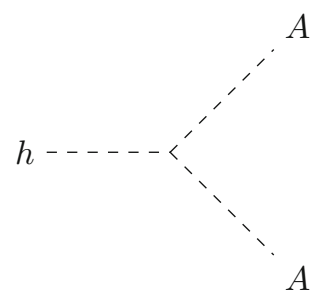

$\lambda_{i}$ basis:

$$
\begin{aligned}
= & i v\left\{\lambda_{1} c_{\beta} s_{\alpha} s_{\beta}^{2}-\lambda_{2} c_{\alpha} c_{\beta}^{2} s_{\beta}+\left(\lambda_{3}+\lambda_{4}\right)\left(c_{\beta}^{3} s_{\alpha}-c_{\alpha} s_{\beta}^{3}\right)\right. \\
& \left.+\lambda_{5}\left(-c_{\beta}^{3} s_{\alpha}+2 c_{\alpha} c_{\beta}^{2} s_{\beta}-2 c_{\beta} s_{\alpha} s_{\beta}^{2}+c_{\alpha} s_{\beta}^{3}\right)\right\} .
\end{aligned}
$$

Physical basis:

$$
\begin{aligned}
= & -\frac{i}{v}\left\{\left(m_{h}^{2}+2 m_{A}^{2}-2 \bar{m}^{2}\right) s_{\beta-\alpha}\right. \\
& \left.+2 \cot 2 \beta\left(m_{h}^{2}-\bar{m}^{2}\right) c_{\beta-\alpha}\right\} .
\end{aligned}
$$

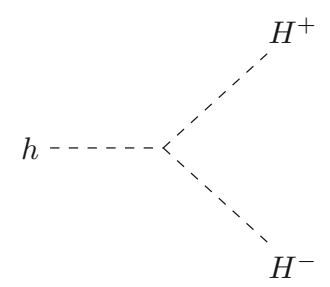

$\lambda_{i}$ basis:

$$
\begin{aligned}
= & i v\left\{\lambda_{1} c_{\beta} s_{\alpha} s_{\beta}^{2}-\lambda_{2} c_{\alpha} c_{\beta}^{2} s_{\beta}+\lambda_{3}\left(c_{\beta}^{3} s_{\alpha}-c_{\alpha} s_{\beta}^{3}\right)\right. \\
& \left.+\left(\lambda_{4}+\lambda_{5}\right)\left(c_{\alpha} c_{\beta}^{2} s_{\beta}-c_{\beta} s_{\alpha} s_{\beta}^{2}\right)\right\} .
\end{aligned}
$$

Physical basis:

$$
\begin{aligned}
= & -\frac{i}{v}\left\{\left(m_{h}^{2}+2 m_{H^{ \pm}}^{2}-2 \bar{m}^{2}\right) s_{\beta-\alpha}\right. \\
& \left.+2 \cot 2 \beta\left(m_{h}^{2}-\bar{m}^{2}\right) c_{\beta-\alpha}\right\} .
\end{aligned}
$$

\section{References}

1. G. Aad et al. [ATLAS Collaboration], Phys. Lett. B 716, 1 (2012). arXiv:1207.7214 [hep-ex]

2. S. Chatrchyan et al. [CMS Collaboration], Phys. Lett. B 716, 30 (2012). arXiv:1207.7235 [hep-ex]

3. G. Aad et al. [ATLAS and CMS Collaborations], JHEP 1608, 045 (2016). arXiv:1606.02266 [hep-ex] 
4. J. de Blas, M. Cepeda, J. D'Hondt, R. Ellis, C. Grojean, B. Heinemann, F. Maltoni, A. Nisati, E. Petit, R. Rattazzi, W. Verkerke, JHEP 01, 139 (2020). arXiv:1905.03764 [hep-ph]

5. J. Alison et al. arXiv:1910.00012 [hep-ph]

6. J.F. Gunion, H.E. Haber, G.L. Kane, S. Dawson, Front. Phys. 80, 1404 (2000). arXiv:hep-ph/9302272 [hep-ph] (SCIPP-89/13. Erratum)

7. M. Aoki, S. Kanemura, K. Tsumura, K. Yagyu, Phys. Rev. D 80, 015017 (2009). arXiv:0902.4665 [hep-ph]

8. G.C. Branco, P.M. Ferreira, L. Lavoura, M.N. Rebelo, M. Sher, J.P. Silva, Phys. Rept. 516, 1 (2012). arXiv:1106.0034 [hep-ph]

9. S.L. Glashow, S. Weinberg, Phys. Rev. D 15, 1958 (1977)

10. N.G. Deshpande, E. Ma, Phys. Rev. D 18, 2574 (1978)

11. G. Bhattacharyya, D. Das, Pramana 87(3), 40 (2016). arXiv:1507.06424 [hep-ph]

12. A.G. Akeroyd, A. Arhrib, E.M. Naimi, Phys. Lett. B 490, 119 (2000). arXiv:hep-ph/0006035

13. A. Barroso, P.M. Ferreira, I.P. Ivanov, R. Santos, JHEP 1306, 045 (2013). arXiv:1303.5098 [hep-ph]

14. P. Bechtle, O. Brein, S. Heinemeyer, G. Weiglein, K.E. Williams, Comput. Phys. Commun. 181, 138 (2010). arXiv:0811.4169 [hep$\mathrm{ph}]$

15. P. Bechtle, O. Brein, S. Heinemeyer, G. Weiglein, K.E. Williams, Comput. Phys. Commun. 182, 2605 (2011). arXiv:1102.1898 [hep$\mathrm{ph}]$

16. P. Bechtle, O. Brein, S. Heinemeyer, O. Stål, T. Stefaniak, G. Weiglein, K.E. Williams, Eur. Phys. J. C 74(3), 2693 (2014). arXiv:1311.0055 [hep-ph]

17. P. Bechtle, S. Heinemeyer, O. Stål, T. Stefaniak, G. Weiglein, Eur. Phys. J. C 75(9), 421 (2015). arXiv:1507.06706 [hep-ph]

18. M. Aaboud et al. [ATLAS Collaboration], Phys. Rev. Lett. 121(19), 191801 (2018). arXiv:1808.00336 [hep-ex] [Erratum: Phys. Rev. Lett. 122 (2019) no.8, 089901]

19. G. Aad et al. [ATLAS Collaboration], Phys. Lett. B 800, 135103 (2020). arXiv:1906.02025 [hep-ex]

20. M. Aaboud et al. [ATLAS Collaboration], Phys. Rev. D 98(5), 052008 (2018). arXiv: 1808.02380 [hep-ex]

21. CMS Collaboration, CMS-PAS-HIG-18-015

22. CMS Collaboration, CMS-PAS-HIG-18-005

23. CMS Collaboration, CMS-PAS-HIG-13-001

24. ATLAS collaboration, ATLAS-CONF-2016-049

25. CMS Collaboration, CMS-PAS-HIG-17-020

26. CMS Collaboration, CMS-PAS-HIG-13-002

27. P. Bechtle, S. Heinemeyer, O. Stål, T. Stefaniak, G. Weiglein, Eur. Phys. J. C 74(2), 2711 (2014). arXiv:1305.1933 [hep-ph]

28. P. Bechtle, S. Heinemeyer, O. Stål, T. Stefaniak, G. Weiglein, JHEP 1411, 039 (2014). arXiv:1403.1582 [hep-ph]

29. References for the individual measurements from the LHC and the Tevatron can be found in: https://higgsbounds.hepforge.org/ downloads.html

30. F. Mahmoudi, Comput. Phys. Commun. 180, 1579 (2009). arXiv:0808.3144 [hep-ph]

31. F. Mahmoudi, Comput. Phys. Commun. 180, 1718-1719 (2009)

32. X.Q. Li, J. Lu, A. Pich, JHEP 1406, 022 (2014). arXiv:1404.5865 [hep-ph]

33. X.D. Cheng, Y.D. Yang, X.B. Yuan, Eur. Phys. J. C 76(3), 151 (2016). arXiv:1511.01829 [hep-ph]

34. P. Arnan, D. Becirevic, F. Mescia, O. Sumensari, Eur. Phys. J. C 77(11), 796 (2017). arXiv:1703.03426 [hep-ph]

35. S. Chen et al. [CLEO], Phys. Rev. Lett. 87, 251807 (2001). arXiv:hep-ex/0108032

36. B. Aubert et al. [BaBar], Phys. Rev. D 77, 051103 (2008). arXiv:0711.4889 [hep-ex]

37. A. Limosani et al. [Belle], Phys. Rev. Lett. 103, 241801 (2009). arXiv:0907.1384 [hep-ex]
38. J. Lees et al. [BaBar], Phys. Rev. Lett. 109, 191801 (2012). arXiv:1207.2690 [hep-ex]

39. J. Lees et al. [BaBar], Phys. Rev. D 86, 052012 (2012). arXiv:1207.2520 [hep-ex]

40. T. Saito et al. [Belle], Phys. Rev. D 91(5), 052004 (2015) arXiv: 1411.7198 [hep-ex]

41. S. Chatrchyan et al. [CMS Collaboration], Phys. Rev. Lett. 111, 101804 (2013). arXiv:1307.5025 [hep-ex]

42. T. Aaltonen et al. [CDF Collaboration], Phys. Rev. D 87(7), 072003 (2013). arXiv:1301.7048 [hep-ex]

43. R. Aaij et al. [LHCb Collaboration], Phys. Rev. Lett. 119(4), 041802 (2017). arXiv: 1704.07908 [hep-ex]

44. M. Aaboud et al. [ATLAS Collaboration], JHEP 04, 098 (2019). arXiv:1812.03017 [hep-ex]

45. M. Tanabashi et al. [Particle Data Group], Phys. Rev. D 98(3), 030001 (2018)

46. M.E. Peskin, T. Takeuchi, Phys. Rev. Lett. 65, 964 (1990)

47. M.E. Peskin, T. Takeuchi, Phys. Rev. D 46, 381 (1992)

48. W. Grimus, L. Lavoura, O.M. Ogreid, P. Osland, J. Phys. G 35, 075001 (2008). arXiv:0711.4022 [hep-ph]

49. G. Funk, D. O’Neil, R.M. Winters, Int. J. Mod. Phys. A 27, 1250021 (2012). arXiv:1110.3812 [hep-ph]

50. D. Eriksson, J. Rathsman, O. Stål, Comput. Phys. Commun. 181, 189 (2010). arXiv:0902.0851 [hep-ph]

51. J. Bernon, J.F. Gunion, H.E. Haber, Y. Jiang, S. Kraml, Phys. Rev. D 92(7), 075004 (2015). arXiv:1507.00933 [hep-ph]

52. A.M. Sirunyan et al. [CMS Collaboration], Eur. Phys. J. C 79(5), 421 (2019). arXiv:1809.10733 [hep-ex]

53. G. Aad et al. [ATLAS Collaboration], Phys. Rev. D 101(1), 012002 (2020). arXiv:1909.02845 [hep-ex]

54. F. Kling, S. Su, W. Su. arXiv:2004.04172 [hep-ph]

55. S. Bertolini, Nucl. Phys. B 272, 77 (1986)

56. W. Hollik, Z. Phys. C 32, 291 (1986)

57. T. Enomoto, R. Watanabe, JHEP 1605, 002 (2016). arXiv:1511.05066 [hep-ph]

58. A. Arbey, F. Mahmoudi, O. Stål, T. Stefaniak, Eur. Phys. J. C 78(3), 182 (2018). arXiv: 1706.07414 [hep-ph]

59. J. Haller, A. Hoecker, R. Kogler, K. Mönig, T. Peiffer, J. Stelzer, Eur. Phys. J. C 78(8), 675 (2018). arXiv:1803.01853 [hep-ph]

60. S. Kraml, T.Q. Loc, D.T. Nhung, L.D. Ninh, Sci. Post Phys. 7(4), 052 (2019). arXiv:1908.03952 [hep-ph]

61. J. Baglio, O. Eberhardt, U. Nierste, M. Wiebusch, Phys. Rev. D 90(1), 015008 (2014). arXiv:1403.1264 [hep-ph]

62. V. Barger, L.L. Everett, C.B. Jackson, A.D. Peterson, G. Shaughnessy, Phys. Rev. D 90(9), 095006 (2014). arXiv:1408.2525 [hep$\mathrm{ph}]$

63. J. Ren, R.Q. Xiao, M. Zhou, Y. Fang, H.J. He, W. Yao, JHEP 1806, 090 (2018). arXiv: 1706.05980 [hep-ph]

64. R. Frederix, S. Frixione, V. Hirschi, F. Maltoni, O. Mattelaer, P. Torrielli, E. Vryonidou, M. Zaro, Phys. Lett. B 732, 142 (2014). arXiv: 1401.7340 [hep-ph]

65. M. Cepeda et al. [CERN Yellow], Rep. Monogr. 7, 221 (2019). arXiv:1902.00134 [hep-ph]

66. S. Di Vita, G. Durieux, C. Grojean, J. Gu, Z. Liu, G. Panico, M. Riembau, T. Vantalon, JHEP 1802, 178 (2018). arXiv:1711.03978 [hep-ph]

67. J. List, Priv. Commun.

68. S. Heinemeyer, B. List, J. List, G. Moortgat-Pick, G. Weiglein, IFT-UAM/CSIC-20-029

69. M.D. Goodsell, F. Staub, Phys. Lett. B 788, 206-212 (2019). arXiv:1805.07310 [hep-ph]

70. N. Chen, T. Han, S. Li, S. Su, W. Su, Y. Wu. arXiv:1912.01431 [hep-ph]

71. G.C. Dorsch, S.J. Huber, J.M. No, JHEP 10, 029 (2013). arXiv:1305.6610 [hep-ph] 
72. G.C. Dorsch, S.J. Huber, K. Mimasu, J.M. No, Phys. Rev. Lett. 113(21), 211802 (2014). arXiv:1405.5537 [hep-ph]

73. G.C. Dorsch, S.J. Huber, K. Mimasu, J.M. No, JHEP 12, 086 (2017). arXiv:1705.09186 [hep-ph]
74. F. Arco, S. Heinemeyer, M.J. Herrero, Work in progress

75. A. Alloul, N.D. Christensen, C. Degrande, C. Duhr, B. Fuks, Comput. Phys. Commun. 185, 2250 (2014). arXiv:1310.1921 [hep-ph] 